\title{
Peuplement des Crustacés dans la zone hyporhéique d'un ruisseau des Pyrénées
}

\author{
R. Rouch 1
}

Mots clés : zone hyporhéique, peuplement des Crustacés, persistance, hétérogénéité.

Le peuplement des Crustacés de la zone hyporhéique d'un ruisseau des Pyrénées, le Nert, a été étudié pendant un an sur une aire de $30 \mathrm{~m}^{2}$ à $0,6 \mathrm{~m}$ et $1 \mathrm{~m}$ de profondeur. 35 espèces dont 20 stygobies ont été recensées. Ce peuplement est caractérisé par sa persistance due à la stabilité physique de l'environnement interstitiel. Il témoigne cependant d'une très forte hétérogénéité liée aux conditions hydrodynamiques qui règnent au sein des sédiments.

The hyporheic crustacean assemblage from a Pyrenean brook

Keywords : hyporheic zone, crustacean assemblage, persistence, heterogeneity.

The hyporheic crustacean assemblage from a $30 \mathrm{~m}^{2}$ area of a Pyrenean brook was studied monthly over one year at a depth of 0,6 and $1 \mathrm{~m} .35$ species with 20 stygobionts were collected. The assemblage persistence of this community is related to the physical stability of the interstitial habitat at this site. However this assemblage has a large spatial heterogeneity globally linked to the substream flow paths.

\section{Introduction}

Au cours des deux dernières décennies, l'intérêt pour le biotope hyporhéique (Orghidan 1959) s'est considérablement accru en raison, notamment, de l'attention que lui ont portée les hydrobiologistes des eaux de surface (Williams \& Hynes 1974, Stanford \& Gaufin 1974, Stanford \& Ward 1988, Williams 1984, 1989, Brestschko 1991). L'image globale qui en est retenue est celle d'un écotone (Gibert et al. 1990) dont l'homogénéité apparente, puisqu'il est limité à l'espace interstitiel (Gibert et al. 1990), cache, en fait, une grande hétérogénéité structurale (Godbout \& Hynes 1982, Danielopol 1983, Danielopol \& Marmonier 1992, Rouch 1988, Boulton et al. 1992, Dole-Olivier et al. 1993) qui retentit sur son peuplement. Les « facteurs synthétiques » (Marmonier \& Dole 1986, Dole-Olivier \& Marmonier 1992) responsables de cette hétérogénéité correspondent aux circulations d'eau en surface et en profondeur

1. Laboratoire souterrain du CNRS, Moulis, F-09200 SaintGirons. d'une part, à la morphologie du lit d'autre part. Danielopol $(1983,1939,1991)$, a particulièrement insisté sur l'aspect dynamique et changeant des assemblages d'individus méiobenthiques dans ce type de milieu.

Contrastant avec ces variations temporelles, les résultats relatifs au milieu hyporhéique d'un ruisseau des Pyrénées, le Lachein (Rouch 1988, 1991, 1992), indiquent au contraire un peuplement persistant, en relation avec la « stabilité physique » (sensu Dole \& Chessel 1986, Marmonier et al. 1992) élevée du site étudié. Cette persistance a été imputée au fait que le ruisseau de Lachein est alimenté par un exutoire karstique (Pospisil 1992, Stanley \& Boulton 1993).

Les recherches entreprises sur un autre ruisseau des Pyrénées, le Nert, ont eu pour but de vérifier si les observations réalisées sur le Lachein constituaient une exception. Le Nert draine un bassin versant composé pour l'essentiel par des séricito-schistes et des pélites à débit schisteux du Silurien et du Carbonifère (Barrouquère 1968) ; son alimentation diffère donc nettement de celle du Lachein. 


\section{Le site étudié}

Il correspond à la station 16 prospectée par Gourbault \& Lescher-Moutoué (1968) qui en avaient montré la richesse du peuplement. Cette station est située à l'aval d'un méandre du Nert, à $425 \mathrm{~m}$ d'altitude et à $700 \mathrm{~m}$ en amont de la confluence de ce cours d'eau avec le Salat. A l'aval de la station, un seuil naturel permet de détourner une partie des eaux vers un moulin (Fig. 1).


L'aire étudiée, arbitrairement divisée en 4 secteurs, A, B, C et D mesure $30 \mathrm{~m}^{2}$. Elle se compose d'un chenal le long de la rive droite (bord des secteurs $B$ et $D$ ) et d'une zone de dépôts qui s'étend de la rive gauche au chenal (Fig. 1). Les caractéristiques physiques, hydrologiques et chimiques de ce site ont été définies dans un autre travail (Rouch et al. sous presse).

La piézométrie (Fig. 1) indique qu'il existe deux axes de drainage : le plus important est établi dans
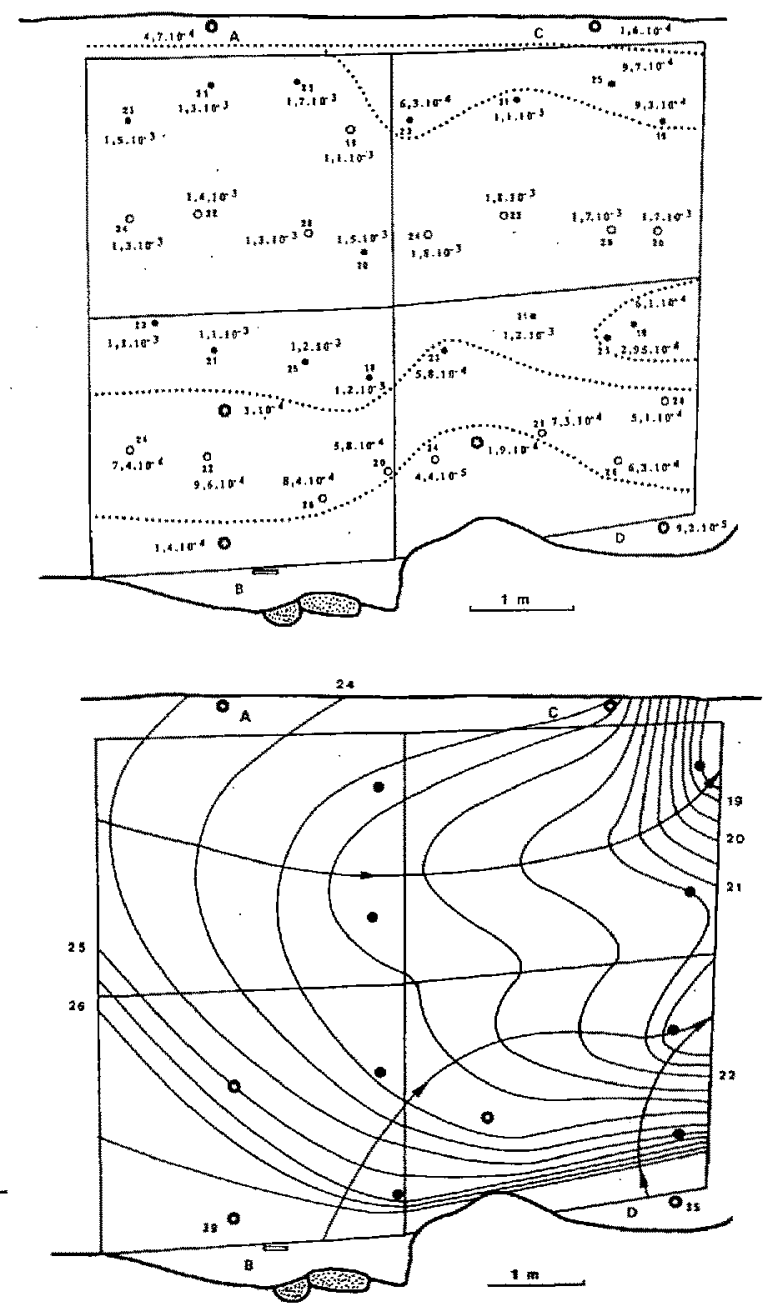

Fig. 1. Le Nert. 1 : emplacement du site étudié ; 2 : topographie du lit du cours d'eau à l'étiagæ ; $3:$ carte piézométrique du site en janvier 1990 (Q ruisseau : $68 \mathrm{l} / \mathrm{s} ; \mathrm{H}$ échelle limnigraphique $=41,5 \mathrm{~cm}$; la hauteur des lignes isopièzes est indiquée en cm par rapport au 0 de l'échelle ; 4 : valeurs de la conductivité hydraulique $(\mathrm{m} / \mathrm{s})$ à $0,6 \mathrm{~m}$ de profondeur (cercles blancs) et à $1 \mathrm{~m}$ de profondeur (cercles noirs).

Fig. 1. Nert brook. $1:$ location of the study site ; $2:$ streambed topography at low discharge ; $3:$ piezometric map of the site in January 1990 (Q stream $=68 \mathrm{l} / \mathrm{s} ; \mathrm{H}$. water-level gauge $=41,5 \mathrm{~cm}$; the height of equipotential lines is expressed in $\mathrm{cm}$ to 0 on the waterlevel gauge); $4:$ hydraulic conductivity values $(\mathrm{m} / \mathrm{s})$ at a depth of $0,6 \mathrm{~m}$ (white dots) and $1 \mathrm{~m}$ (black dots). 

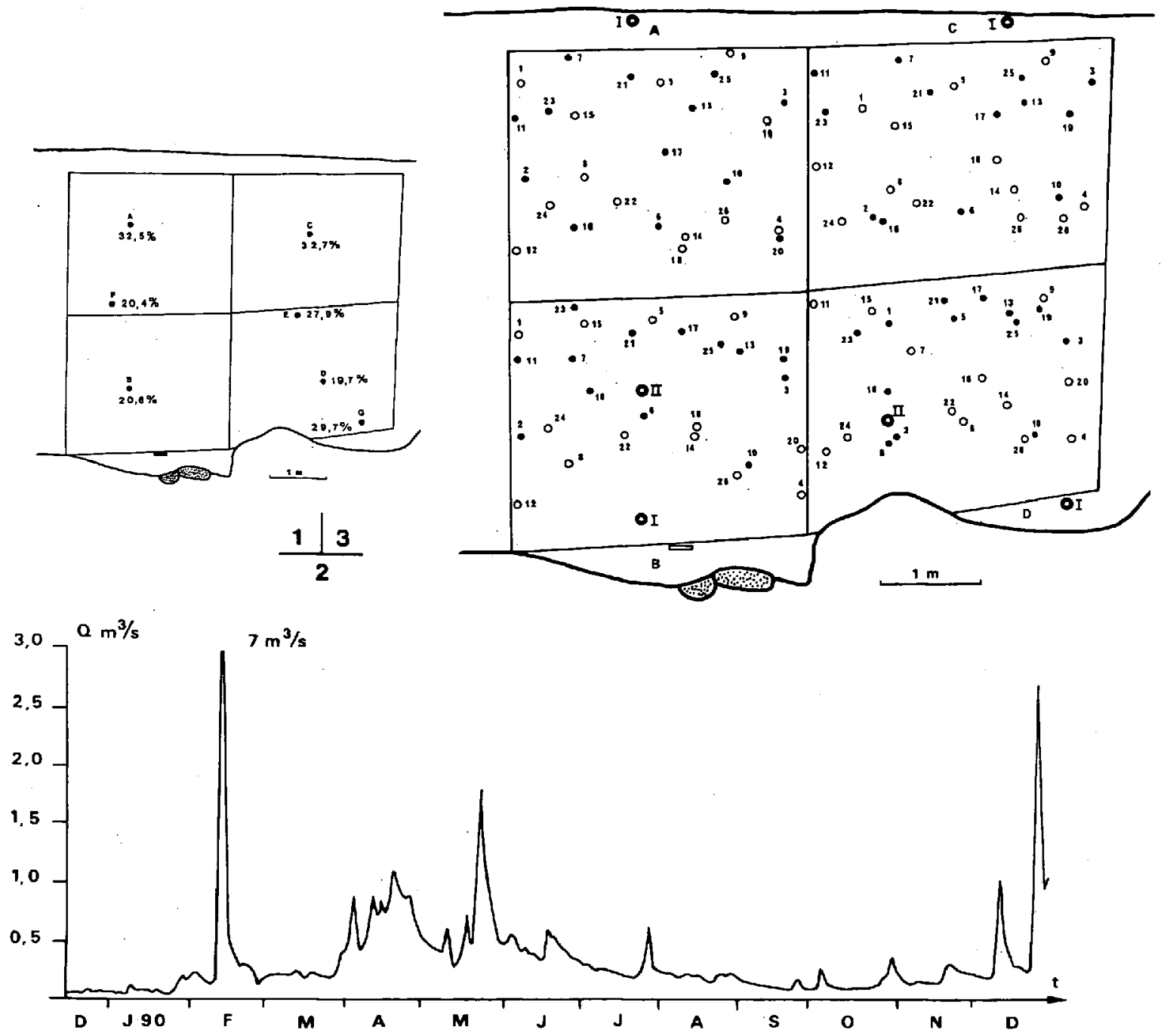

Fig. 2. Le Nert. 1 : valeurs de la porosité ; 2 : débits du Nert de décembre 1989 à décembre $1990 ; 3$ : emplacement des mini-piezomètres fixes (AI, BI, CI, DI, BII, DII) et des points de prélèvements (cercles blancs $=0,6 \mathrm{~m}$ de profondeur ; cercles noirs $=1 \mathrm{~m}$ de profondeur).

Fig. 2. Nert brook. 1 : porosity values ; 2 : Nert flow rates from December 1989 to December $1990 ; 3:$ location of fixed mini-piezometers (AI, BI, CI, DI, BII, DII) and of sampling points (white dots $=0,6 \mathrm{~m}$ deep ; black dots $=1 \mathrm{~m}$ deep).

le banc de graviers, le long des secteurs $\mathrm{A}$ et $\mathrm{C}$; le moins développé apparaît sous le secteur $D$. Les valeurs de la conductivité hydraulique (Fig. 1) sont comprises entre $1.10^{-4} \mathrm{~m} / \mathrm{s}$ et $5: 10^{-4} \mathrm{~m} / \mathrm{s}$ à proximité des rives, $1.10^{-3} \mathrm{~m} / \mathrm{s}$ et $2.10^{-3} \mathrm{~m} / \mathrm{s}$ dans le banc de gravier et $5.10^{-4} \mathrm{~m} / \mathrm{s}$ et $9.10^{-4} \mathrm{~m} / \mathrm{s}$ sous le chenal. La porosité (Fig. 2) est plus élevée dans le banc de graviers $(32,5 \%)$ que sous le chenal $(20 \%)$.

La période étudiée s'étend de décembre 1989 à décembre 1990. Elle fait suite à une année exception- nellement sèche. C'est pourquoi un régime de basses eaux prononcé caractérise la fin de 1989 ainși que les trois premiers mois de l'année suivante, interrompu seulement par une forte crue en février (Fig. 2). D'avril à août, une période de hautes eaux s'installe suivie par un étiage estival et automnal (septembre et octobre) auquel succède une légère recrudescence des débits en novembre et décembre. Durant toute l'année, le secteur étudié s'est comporté comme une zone de recharge (Rouch et al., sous presse). 


\section{Matériel et méthodes}

La technique utilisée est celle des sondages tubés (Bou \& Rouch 1967). Chaque mois, 8 échantillons (2 par secteurs) repérés par leur coordonnées (Fig. 2) ont été prélevés à deux profondeurs différentes (4 à $0,6 \mathrm{~m}$ et 4 à $1 \mathrm{~m}$ ). Les sondes étaient déplacées lors de chaque série de prélèvements afin d'éviter les modifications entraînées par des pompages successifs sur des sondes fixes (Gibert et al. 1981, Dole 1983). Chaque prélèvement correspond à un pompage de 10 litres dont le résidu solide, filtré sur un filet de $110 \mu \mathrm{m}$ de maille, a été fixé dans l'alcool à $70^{\circ}$. Les échantillons obtenus sont caractérisés par la lettre du secteur dont ils sont issus et par un numéro : 1-2 (décembre 1989), 3-4 (janvier 1990)... 25-26 (décembre 1990). Ils ont été prélevés pour les débits ponctuels suivants : $18.12 .89=57 \mathrm{l} / \mathrm{s}$; $17.1 .90=68 \mathrm{l} / \mathrm{s} ; 19.2 .90=364 \mathrm{l} / \mathrm{s} ; 13.3 .90=$ $146 \mathrm{l} / \mathrm{s} ; 17.4 .90=890 \mathrm{l} / \mathrm{s} ; 14.5 .90=310 \mathrm{l} / \mathrm{s}$; $11.6 .90=460 \mathrm{l} / \mathrm{s} ; 10.7 .90=364 \mathrm{l} / \mathrm{s} ; 7.8 .90=$ $202 \mathrm{l} / \mathrm{s} ; 17.9 .90=46 \mathrm{l} / \mathrm{s} ; 16.10 .90=85 \mathrm{l} / \mathrm{s}$; $12.11 .90=165 \mathrm{l} / \mathrm{s} ; 10.12 .90=180 \mathrm{l} / \mathrm{s}$.

A partir de juillet 1990, deux sondes fixes $\mathrm{H}$ et J (Fig. 1) ont été implantées dans la nappe alluviale de la rive gauche. Les échantillons, prélevés comme précédemment, ont été numérotés de la façon suivante : H15 et J15 (juillet 1990), H17 et J17 (août 1990)... H25 et J25 (décembre 1990).

Au total, 116 prélèvements ont été réalisés, 104 dans le milieu hyporhéique et 12 dans la nappe. Après coloration au rose bengale, les Crustacés ont été triés dans leur totalité, soit 21719 individus provenant du milieu hyporhéique (dont 21628 ont été déterminés, l'échantillon $\mathrm{C} 2$ des Cyclopides ayant été détruit) et 509 de la nappe.

\section{Caractéristiques globales du peuplement}

\subsection{Richesse spécifique}

36 espèces de Crustacés ont été dénombrées dont 21 stygobies (Tableau 1). Le groupe le plus diversifié est celui des Copépodes (7 espèces de Cyclopides, 17 d'Harpacticides) suivi par les Amphipodes (5 espèces), les Ostracodes ( 4 espèces), les Isopodes ( 2 espèces) et les Syncarides ( 1 espèce). Le milieu hyporhéique avec 35 espèces dont 20 stygobies, est plus riche que la nappe où 21 espèces (14 stygobies) seulement ont été mises en évidence. Cette diminution de la richesse spécifique concerne non seulement les espècess épigées mais aussi les stygobies, observation qui doit cependant être tempérée du fait que seules 2 stations de la nappe ont été prospectées.

La similarité faunistique de ces deux milieux n'en est pas moins élevée : le coefficient de Sørensen a pour valeur $0,714(\mathrm{Sr}=0,764$ pour les espèces stygobies et $\mathrm{Sr}=0,636$ pour les espèces épigées).

\subsection{Abondance}

Dans le milieu hyporhéique, les Copépodes avec 96,8\% des captures (Tableau 2) sont les Crustacés les plus abondants et, notamment, les Cyclopides qui représentent $72,7 \%$ des individus récoltés. Il n'existe pas de différence significative de l'abondance de l'ensemble des Crustacés entre les 13 séries de prélèvements (test de Kruskal-Wallis, $H=14,19$ pour $\chi^{2} 0,05=21,03$ avec $12 \mathrm{dl}$ ). De ce fait, aucune liaison n'apparaît entre l'abondance des individus récoltés et les valeurs de débit du cours d'eau (Fig. 3). La densité moyenne des 104 prélèvements est égale à $209 \pm 31 \mathrm{i} / 10 \mathrm{l}$ (seuil de $5 \%$ ). Toutefois, les densités à 0,6 et à $1 \mathrm{~m}$ de profondeur diffèrent de façon significative (test de Mann-Whitney, $\mathrm{Z}=4,39$ avec $\mathrm{p}$ associée $=0,00003$ ) ; la densité moyenne à $0,6 \mathrm{~m}$ est de $279 \pm 54 \mathrm{i} / 10 \mathrm{l}$ (seuil de $5 \%$ ), celle à $1 \mathrm{~m} \mathrm{de:} 138 \pm 19 \mathrm{i} / 10 \mathrm{l}$ (seuil de $5 \%$ ). La répartition des densités n'est pas homogène (Fig. 4) avec des valeurs plus faibles sous le chenal et sous le dôme piézométrique médian quelle que soit la profondeur.

Il faut noter enfin que le rang selon leur abondance des 35 espèces de Crustacés du milieu hyporhéique lors des 13 séries de prélèvements (Tableau 3) ne montre pas de différence significative (coefficient de concordance de rang de Kendall $W=0,840$ et $\chi^{2}=371,28$ avec $\chi^{2} 0,005=58,96$ pour $34 \mathrm{dl}$ ).

Dans la nappe (Tableau 2), les Cyclopides demeurent les plus abondants $(39,7 \%)$ suivis par les Amphipodes $(38,7 \%)$. Les densités des six séries de prélèvements sur l'ensemble des deux sondes ne diffèrent pas de façon significative $(\mathrm{H}$ de Kruskal Wallis $=5,32$ pour $\chi^{2} 0,05=11,07$ avec $5 \mathrm{dl}$ ) ; la densité moyenne des 12 prélèvements est égale à 42 $\pm 16 \mathrm{i} / 101$ (au seuil de $5 \%$ ). Toutefois, les densités au niveau de la sonde $\mathrm{H}$ diffèrent de façon significative de celles observées en $J$ (test $U$ de MannWhitney : $Z=4$ avec $\mathrm{p}$ associée $=0,013$ ); la 
Tableau 1. Liste des Crustacés récoltés : Ostracodes (dét. Danielopol), Cyclopides (dết. Lescher-Moutoué), Syncarides (dét. Coineau), Isopodes (dét. Magniez et Coineau), Amphipodes (dét. Bou). Les espèces stygobies sont indiquées par un astérique.

Table 1. Crustacea collected from interstitial biotopes of the Nert brook ; ostracods (det. Danielopol), cyclopoids (det. Lescher-Moutoué), syncarids (det. Coineau), isopods (det. Magniez and Coineau), amphipods (det. Bou). The stygobiont species are indicated with an asterisk.




Tableau 2. Nombre total de Crustacés récoltés et leur pourcentage.

Table 2. Total number of Crustacea collected with their relative abundance.

\begin{tabular}{|c|c|c|c|c|c|c|c|}
\hline \multirow{2}{*}{ Groupes } & \multirow{2}{*}{ Ost. } & \multicolumn{2}{|c|}{ Copepodes } & Sync. & Isop. & Amphi. & Total \\
\cline { 3 - 7 } & & Cycl. & Harp. & & & & \\
\hline Mil. hypor. & 71 & 15783 & 5240 & 94 & 142 & 389 & 21719 \\
& $0,3 \%$ & $72,7 \%$ & $24,1 \%$ & $0,4 \%$ & $0,7 \%$ & $1,8 \%$ & \\
\hline Nappe & 32 & 202 & 34 & 26 & 18 & 197 & 509 \\
& $6,3 \%$ & $39,7 \%$ & $6,7 \%$ & $5,1 \%$ & $3,5 \%$ & $38,7 \%$ & \\
\hline
\end{tabular}

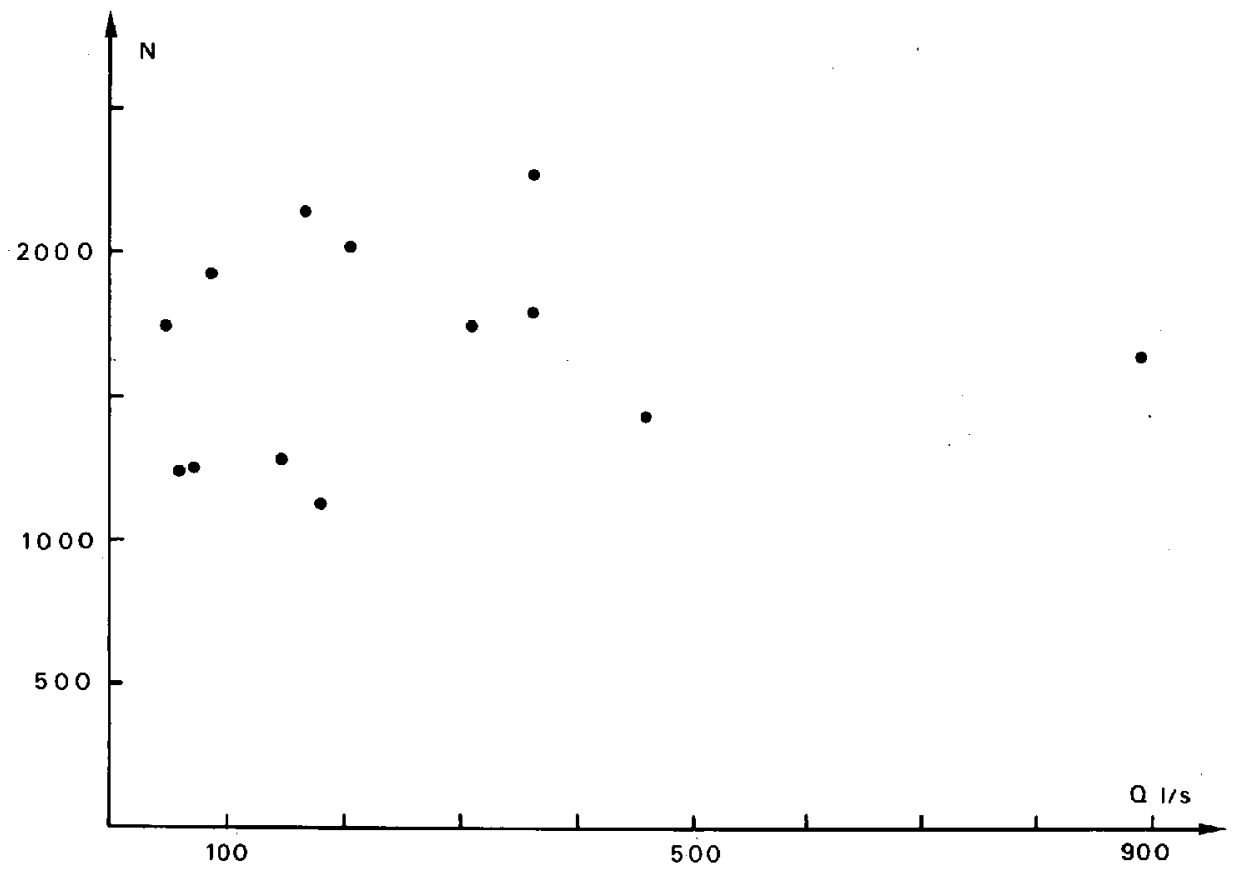

Fig. 3. Nombres de Crustacés capturés et valeurs des débits du Nert lors des 13 séries de prélèvements.

Fig. 3. Numbers of Crustacea collected and Nert flow rates values for the 13 monthly samples series.
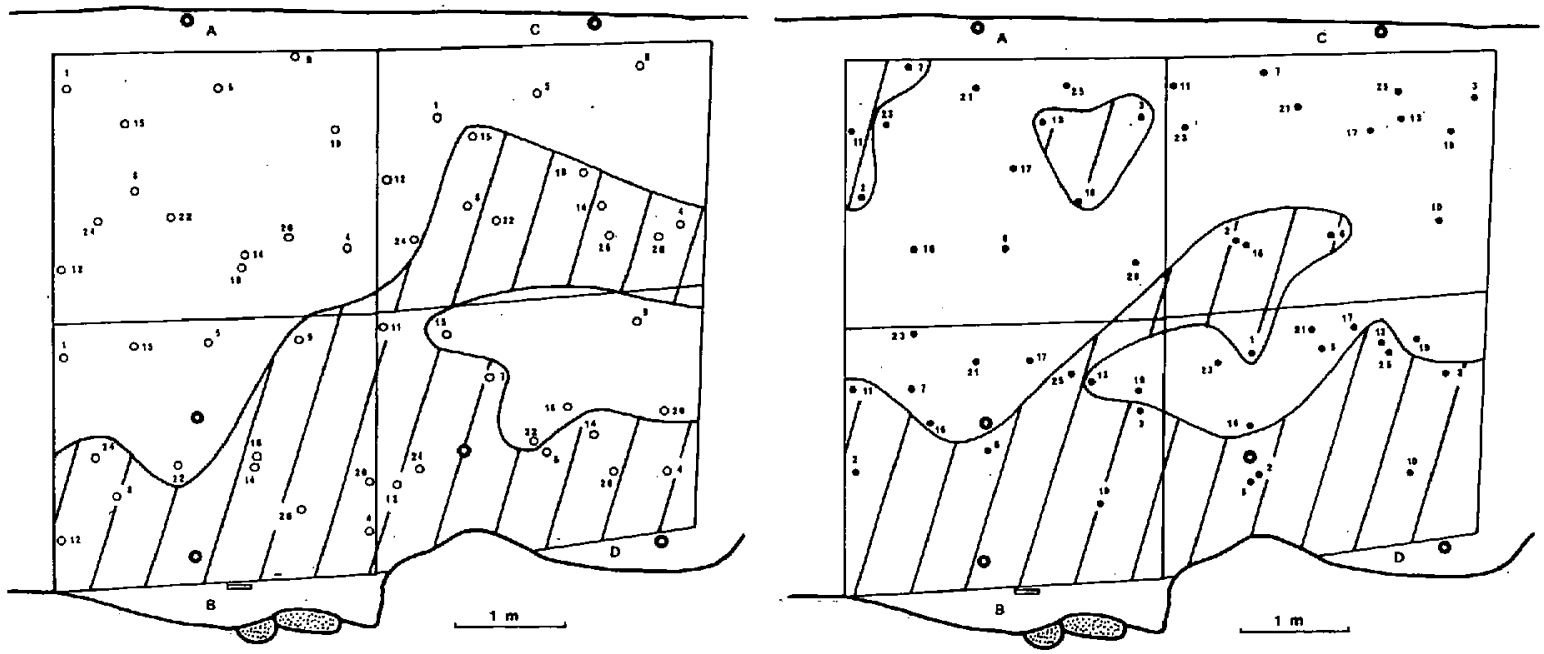

Fig. 4. Distribution des densités des Crustacés à $0,6 \mathrm{~m}$ (cercles blancs) et à $1 \mathrm{~m}$ (cercles noirs); les aires hachurées correspondent à des valeurs respectivement inférieures à $220 \mathrm{i} / 10 \mathrm{l}$ et à $110 \mathrm{i} / 10 \mathrm{l}$.

Fig. 4. Distribution of crustacean densities at a depth of $0,6 \mathrm{~m}$ (white dots) and $1 \mathrm{~m}$ (black dots); the hatched areas have respective values below $220 \mathrm{i} / 10 \mathrm{l}$ and $110 \mathrm{i} / 10 \mathrm{l}$. 
Tableau 3. Zone hyporhéique. Nombre d'individus récoltés lors de chaque série de prélèvements.

Table 3. Hyporheic zone. Number of animals collected monthly.

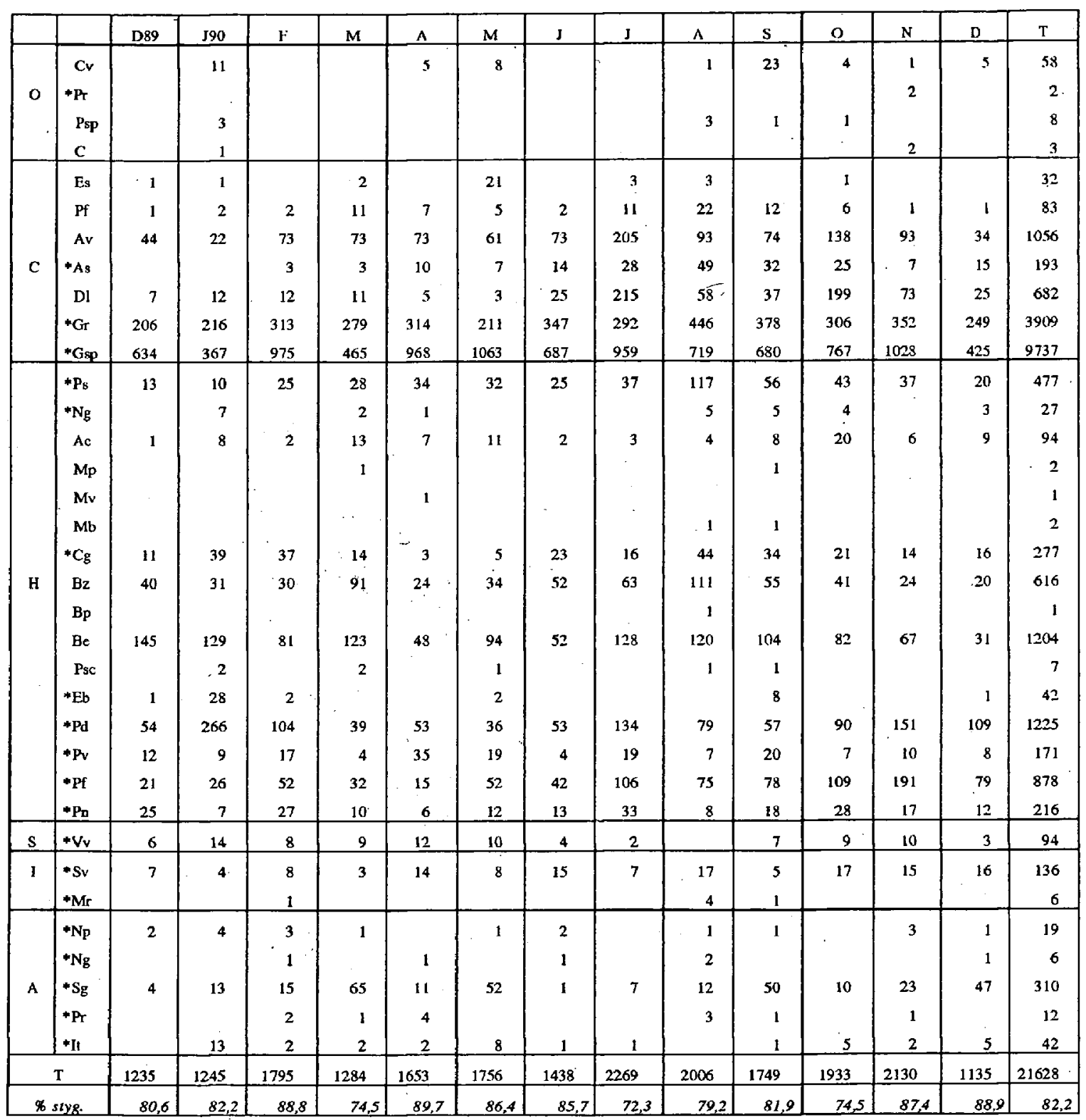

densité moyenne en $\mathrm{H}$ est de $59 \pm 10 \mathrm{i} / 10 \mathrm{l}$ (seuil de $5 \%$ ) et celle en J de $26 \pm 26 \mathrm{i} / 101$ (seuil de $5 \%$ ). Comme pour la zone hyporhéique, le rang selon leur abondance des 21 espèces récoltées lors des 6 séries de prélèvements (Tableau 4) n'indique pas de différence significative ( $W$ de Kendall $=0,596$ et $\chi^{2}=71,5$ avec $\chi^{2} 0,001=45,32$ pour $20 \mathrm{dl}$ ).

\subsection{Espèces stygobies et épigées}

Les espèces stygobies sont largement majoritaires dans les deux milieux puisqu'elles représentent $82,2 \%$ et $86,8 \%$ des Crustacés respectivement récoltés dans la zone hyporhéique et dans la nappe.

Dans le milieu hyporhéique, le pourcentage des stygobies ne change pratiquement pas avec la 
Tableau 4. Nappe alluviale. Nombre d'individus récoltés lors de chaque série de prélèvements.

Table 4. Alluvial groundwater. Number of animals collected monthly.

\begin{tabular}{|c|c|c|c|c|c|c|c|c|}
\hline & & J 90 & $\mathrm{~A}$ & $S$ & 0 & $\mathbf{N}$ & D & $\mathrm{T}$ \\
\hline 0 & $\begin{array}{l}\mathrm{Cv} \\
{ }^{*} \mathrm{Pr} \\
\text { Psp } \\
\text { C ind. }\end{array}$ & $\begin{array}{l}2 \\
4\end{array}$ & 7 & 4 & $\begin{array}{l}5 \\
1 \\
1\end{array}$ & 1 & 7 & $\begin{array}{r}2 \\
27 \\
1 \\
2 \\
\end{array}$ \\
\hline C & $\begin{array}{l}\text { Av } \\
\text { Dl } \\
* \text { Gr } \\
{ }^{*} \mathrm{Gsp} \\
\end{array}$ & $\begin{array}{r}23 \\
17 \\
\end{array}$ & $\begin{array}{r}3 \\
31 \\
\end{array}$ & $\begin{array}{r}3 \\
41 \\
\end{array}$ & $\begin{array}{r}1 \\
1 \\
35 \\
\end{array}$ & $\begin{array}{r}1 \\
3 \\
14 \\
\end{array}$ & $\begin{array}{r}10 \\
2 \\
17 \\
\end{array}$ & $\begin{array}{r}1 \\
40 \\
6 \\
155 \\
\end{array}$ \\
\hline $\mathbf{H}$ & $\begin{array}{l}\mathrm{Be} \\
\mathrm{Bz} \\
* \mathrm{~Eb} \\
* \mathrm{Ach} \\
{ }^{*} \mathrm{Pv} \\
{ }^{*} \mathrm{Pfm} \\
{ }^{*} \mathrm{Pn} \\
\end{array}$ & $\begin{array}{l}1 \\
4\end{array}$ & $\begin{array}{l}1 \\
1\end{array}$ & $\begin{array}{l}4 \\
4 \\
1 \\
1\end{array}$ & $\begin{array}{l}5 \\
4 \\
1 \\
2 \\
1\end{array}$ & 1 & 2 & $\begin{array}{r}10 \\
11 \\
5 \\
3 \\
3 \\
1 \\
1\end{array}$ \\
\hline$S$ & ${ }^{*} \mathrm{Vv}$ & & 4 & 4 & 4 & 9 & 5 & 26 \\
\hline I & $\begin{array}{l}{ }^{*} \mathrm{~Sv} \\
{ }^{*} \mathrm{Mr}\end{array}$ & & 2 & 4 & $\begin{array}{l}1 \\
1 \\
\end{array}$ & $\begin{array}{l}1 \\
3 \\
\end{array}$ & 6 & $\begin{array}{r}4 \\
14 \\
\end{array}$ \\
\hline A & $\begin{array}{l}{ }^{*} \mathrm{~Np} \\
{ }^{*} \mathrm{Sg} \\
{ }^{*} \mathrm{Pr} \\
\end{array}$ & $\begin{array}{l}2 \\
2\end{array}$ & $\begin{array}{l}2 \\
2\end{array}$ & $\begin{array}{l}2 \\
8 \\
1 \\
\end{array}$ & $\begin{array}{r}9 \\
47\end{array}$ & $\begin{array}{l}13 \\
49\end{array}$ & $\begin{array}{r}6 \\
54\end{array}$ & $\begin{array}{r}34 \\
162 \\
1 \\
\end{array}$ \\
\hline & $\mathrm{T}$ & 56 & 53 & 77 & 119 & 95 & 109 & 509 \\
\hline & styg. & 53,6 & 90,6 & 85,7 & 89,1 & 96,8 & 90,8 & 86,8 \\
\hline
\end{tabular}

profondeur. En effet, pour les 13 séries de prélèvements, sa valeur moyenne est égale à $82 \%$ à $0,6 \mathrm{~m}$ de profondeur ( $\mathrm{cv}=9,9 \%$ ) et à $81,3 \%$ à $1 \mathrm{~m}$ de profondeur $(\mathrm{cv}=13,7 \%)$.

\section{Le peuplement de la zone hyporhéique}

\subsection{Richesse spécifique}

Lors de chacune des 13 séries d'échantillons, la richesse spécifique totale (Fig. 5 et Tableau 3) a varié entre 20 et 29 espèces (moyenne $=24 \pm 1,6$ au seuil de $5 \%$ ). La similarité faunistique entre ces 13 séries d'échantillons comparées deux à deux reste toujours élevée, la valeur moyenne du coefficient de Sørensen s'établissant à $0,858(\mathrm{n}=78, \mathrm{cv}=5,3 \%)$.

La richesse spécifique moyenne par prélèvement dans chacune de ces 13 séries, nettement plus basse (Fig. 5), diffère de façon très significative ( $F$ de Fisher $=4,19$ pour dl $1=12$ et dl2 $=91$ ). Les valeurs de février, août, septembre et octobre sont plus élevées que celles des autres mois. Sur l'ensemble des échantillons, la richesse spécifique moyenne par prélèvement est de $12,2 \pm 0,6$ (au seuil de $5 \%$ ); elle est égale à $12,8 \pm 0,7(5 \%)$ à $0,6 \mathrm{~m}$ de profondeur et à $11,5 \pm 1(5 \%)$ à $1 \mathrm{~m}$ de profondeur. Bien que ces deux dernières valeurs soient proches, elles diffèrent significativement ( $\mathrm{t}$ de Student $=2,05$ pour $102 \mathrm{dl}$ ).

\subsection{Fréquence d'occurence et répartition des espèces}

Parmi les 35 espèces recensées, 16 peuvent être considérées comme rares avec des fréquences d'occurence inférieures ou égales à $12,5 \%$ (Tableau 5 ). Les 19 autres, beaucoup plus communes (18 à $96 \%$ ), présentent trois types de répartition sur le site. 13 espèces occupent l'ensemble de l'aire étudiée : les Cyclopides Graeteriella sp. (Fig. 6), G. rouchi, $P$. fimbriatus, $D$. languidoides, $A$. venustus, $A$. sensitivus, les Harpacticides $P$. subterranea, $P$. nertensis, $P$. $f$ meridionalis, $P$. dianae, $B$. echinatus,

Tableau 5. Zone hyporhéique. Nombre de stations par espèces à $0,6 \mathrm{~m}$ et $1 \mathrm{~m}$ de profondeur.

Table 5. Hyporheic zone. Number of stations for species at a depth of $0,6 \mathrm{~m}$ and $1 \mathrm{~m}$.

\begin{tabular}{|c|c|c|c|c|c|c|}
\hline & \multicolumn{2}{|c|}{ nh, stations } & \multirow[t]{2}{*}{ Total } & \multirow[t]{2}{*}{$\%$} & \\
\hline & & $0,6 \mathrm{~m}$ & $1 \mathrm{~m}$ & & & \\
\hline \multirow{4}{*}{ Ostr. } & $\mathrm{Cv}$ & 12 & 1 & 13 & 12,5 & \\
\hline & ${ }^{*} \operatorname{Pr}$ & 1 & & 1 & 0,9 & \\
\hline & Psp & 4 & 2 & 6 & 5,7 & \\
\hline & C. Ind. & 2 & & 2 & 1,9 & \\
\hline \multirow{7}{*}{ Cycl. } & Es & 4 & 4 & 8 & 7,7 & \\
\hline & Pf & $25 *$ & 12 & 37 & 35,6 & $\mathbf{s}$ \\
\hline & Av & 48 & 44 & 92 & 88,5 & n.s \\
\hline & ${ }^{*}$ As & 27 & 20 & 47 & 45,2 & $\mathrm{n} \cdot \mathrm{s}$ \\
\hline & Dl & $41^{*}$ & 22 & 63 & 60,6 & $\mathbf{s}$ \\
\hline & ${ }^{*} \mathrm{Gr}$ & 49 & 48 & 97 & 93,3 & n.s \\
\hline & *Gsp & 51 & 49 & 100 & 96,2 & n.s \\
\hline \multirow{16}{*}{ Harp. } & ${ }^{*} \mathrm{Ps}$ & 35 & 31 & 66 & 63,5 & n.s \\
\hline & ${ }^{*} \mathrm{Ng}$ & 10 & 1 & 11 & 10,6 & \\
\hline & $A C$ & 19 & 16 & 35 & 33,7 & $\pi . s$ \\
\hline & $\mathrm{Mp}$ & 2 & & 2 & 1,9 & \\
\hline & Mv & 1 & & 1 & 0,9 & \\
\hline & $\mathrm{Mb}$ & 2 & & 2 & 1,9 & \\
\hline & ${ }^{*} \mathrm{Cg}$ & 10 & $33^{*}$ & 43 & 41,3 & $\mathbf{s}$ \\
\hline & $\mathrm{Bz}$ & 50 & 45 & 95 & 91,3 & n.s \\
\hline & $\mathbf{B p}$ & 1 & & 1 & 0,9 & \\
\hline & $\mathrm{Be}$ & 50 & 47 & 97 & 93,3 & $\mathrm{n}, \mathrm{s}$ \\
\hline & Psc & 7 & & 7 & 6.7 & \\
\hline & $* \mathrm{~Eb}$ & 3 & 5 & 8 & 7,7 & \\
\hline & *Pd & $45^{*}$ & 27 & 72 & 69,2 & $\mathrm{~s}$ \\
\hline & ${ }^{*} \mathrm{Pv}$ & 24 & 19 & 43 & 41,3 & n.s \\
\hline & *Pfm & $46^{*}$ & 26 & 72 & 69,2 & $\mathrm{~s}$ \\
\hline & *Pn & 31. & 31 & 62 & 59,6 & n.s \\
\hline Sync. & $* \mathrm{Vy}$ & 12 & $25^{*}$ & 37 & 35,6 & $\mathrm{~s}$ \\
\hline \multirow[t]{2}{*}{ Isop. } & ${ }^{*} \mathrm{~Sv}$ & 28 & 32 & 60 & 57,7 & n.s. \\
\hline & ${ }^{*} \mathrm{Mr}$ & 1 & 3 & 4 & 3,8 & \\
\hline \multirow{5}{*}{ Amph. } & $* \mathrm{~Np}$ & 9 & 4 & 13 & 12,5 & \\
\hline & ${ }^{*} \mathrm{Ng}$ & 2 & 3 & 5 & 4,8 & \\
\hline & ${ }^{*} \mathrm{Sg}$ & 13 & $27^{*}$ & 40 & 38,5 & $\mathbf{s}$ \\
\hline & $* \mathrm{Pr}$ & 3 & 8 & 11 & 10,6 & \\
\hline & ${ }^{*}$ It & 12 & 7 & 19 & 18,3 & n.s \\
\hline
\end{tabular}




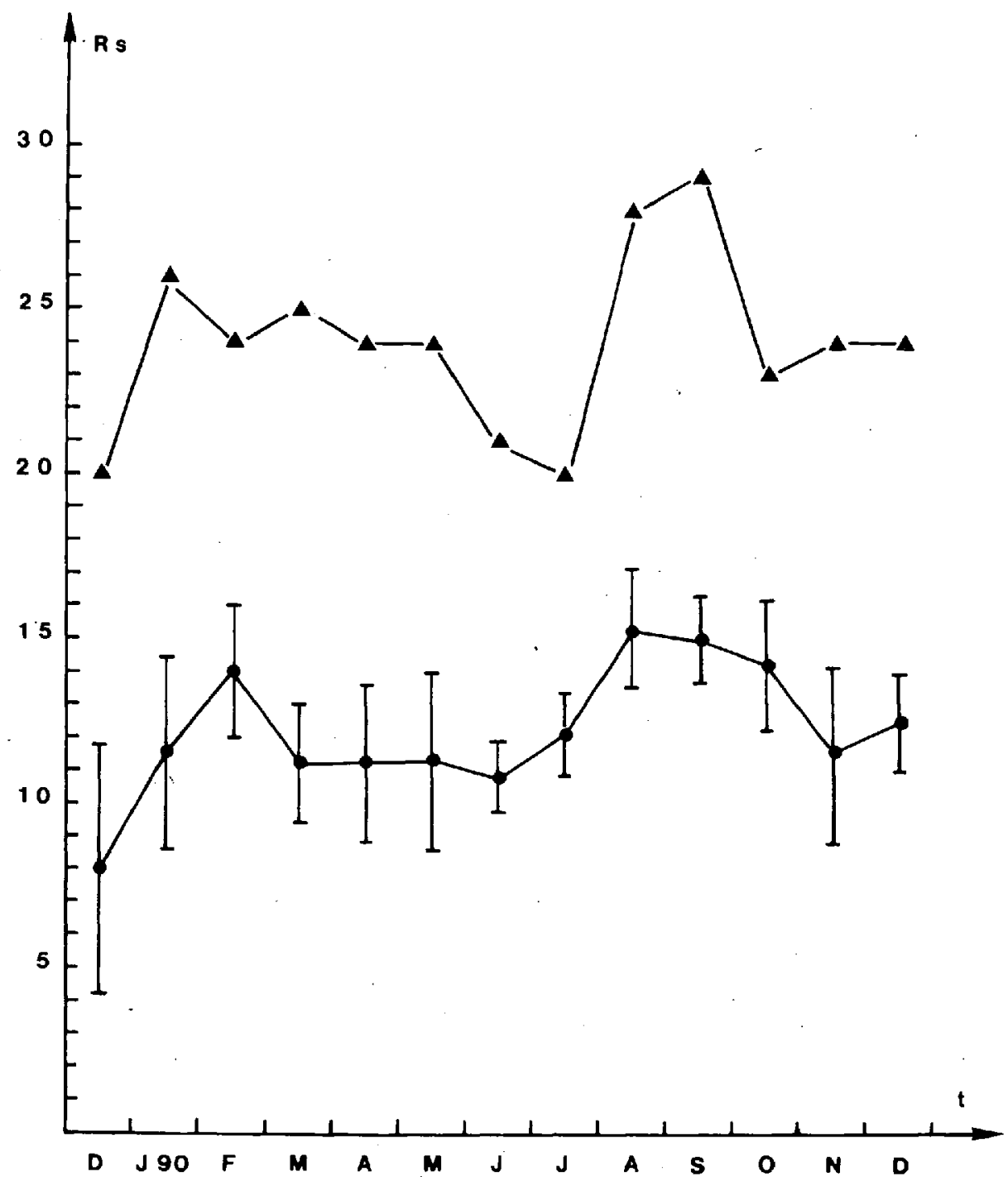

Fig. 5. Richesse spécifique totale (triangles noirs) et richesse spécifique moyenne par prélèvement (cercles noirs) lors des 13 séries d'échantillons.

Fig. 5. Total species richness (black triangles) and average sample value of the species richness (black dots) for the 13 samples series.

B. zschokkei et l'Isopode S. virei boui. 2 espèces sont limitées au banc de graviers, les Harpacticides C. gallicus (Fig. 6) et P. vandeli (Fig. 6). 4 espèces occupent préférentiellement les zones à plus faibles perméabilité (sous le chenal et à proximité de la rive gauche), l'Harpacticide $A$. crassa, le Syncaride $V$. vandeli, les Amphipodes $S$. gineti et $I$. thibaudi (Fig. 6).
Parmi ces 19 espèces communes, 4 sont significativement plus fréquentes (test d'indépendance fondé sur l'écart normal) à $0,6 \mathrm{~m}$ de profondeur qu'à $1 \mathrm{~m}$ (Tableau 5): 2 épigées $P$. fimbriatus et $D$. languidoides, 2 stygobies, $P$. dianae et $\mathrm{P}$. $f$. meridionalis ; 3 espèces stygobies sont significativement plus fréquentes à $1 \mathrm{~m}$ de profondeur : C. gallicus (Fig. 6), $V$. vandeli et $S$. gineti. 

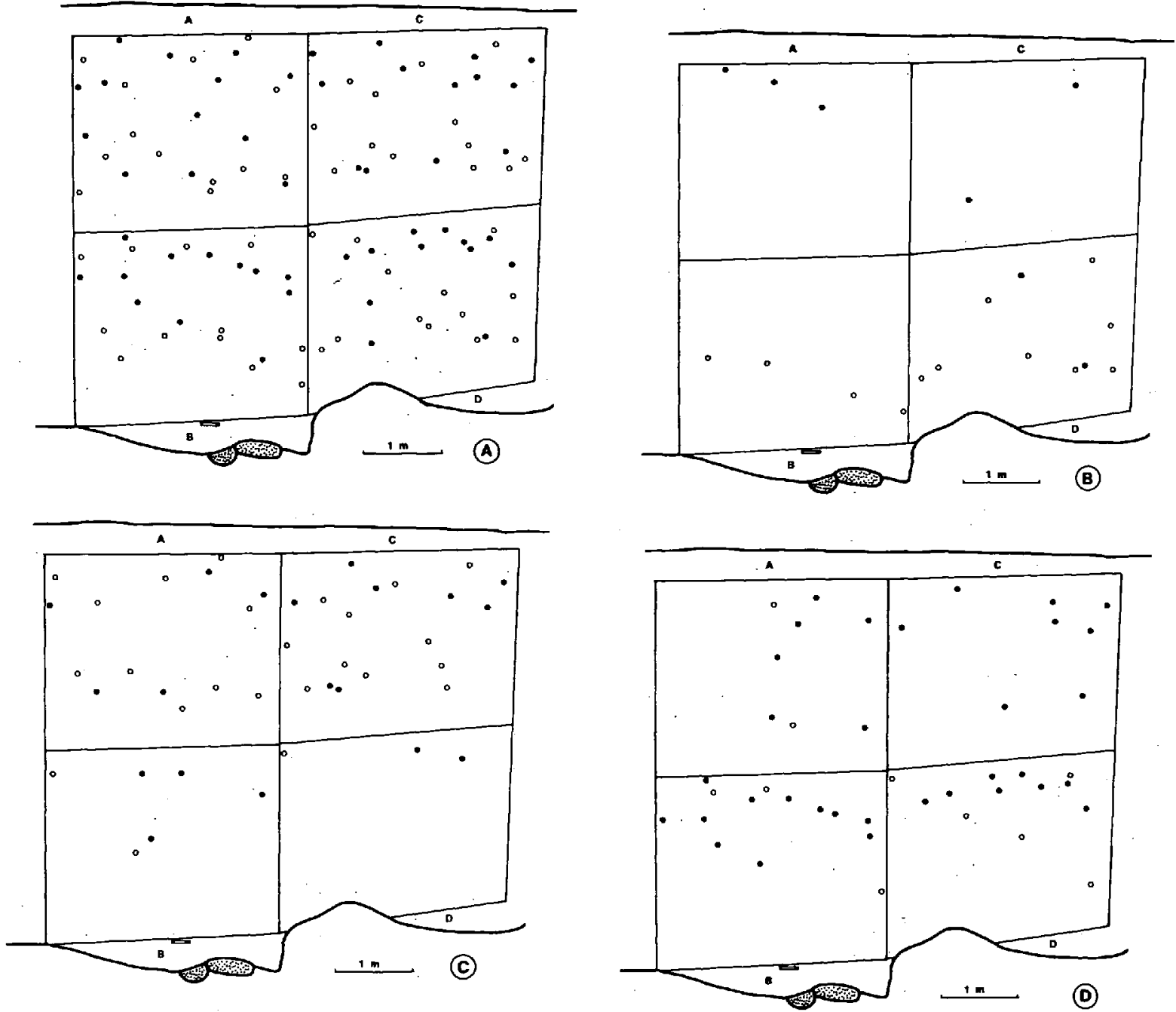

Fig. 6. Répartition spatiale de Graeteriella sp. (A), Ingolfiella thibaudi (B.), Parastenocaris vandeli (C) et Ceuthonectes gallicus (D) ; les cercles blancs indiquent les stations a $0,6 \mathrm{~m}$ de profondeur, les cercles noirs celles a $1 \mathrm{~m}$ de profondeur.

Fig. 6. Spatial distribution of Graeteriella sp. (A), Ingolfiella thibaudi (B), Parastenocaris vandeli (C) and Ceuthonectes gallicus (D) ; $0,6 \mathrm{~m}$ deep samples are indicated with white dots, $1 \mathrm{~m}$ deep samples with black dots.

\subsection{Abondance des espèces}

16 des 19 espèces les plus fréquentes (cf. ante) sont présentes dans les 13 séries de prélèvements. Les 3 autres, $A$. sensitivus (11/13), I. thibaudi (11/13), $V$. vandeli $(12 / 13)$ font défaut dans une ou deux séries.

Ces 19 espèces sont également les plus abondantes (tableau 3). A l'exception de B. zschokkei, ces espèces ne présentent pas de différence significative de leur abondance si l'on compare les différentes séries de prélèvements. En effet, pour chacune des espèces constantes, le $\mathrm{H}$ de Kruskal-Wallis est inférieur à $X^{2}=21,03(\alpha=0,05$ et $12 \mathrm{dl})$; il est également inférieur à $\chi^{2}=19,68(\alpha=0,05$ et $11 \mathrm{dl})$ pour $V$. vandeli et à $X^{2}=18,31(\alpha=0,05$ et $10 \mathrm{dl})$ pour $A$. sensitivus et $I$. thibaudi. Pour 12 de ces espèces (Tableau 6), il n'existe également pas de différence significative de leur abondance à 0,6 et $1 \mathrm{~m}$ de profondeur (U de Mann-Whitney). En revanche, les espèces stygobies Graeteriella sp., $P$. dianae, 
Tableau 6. Zone hyporhéique. Nombre total d'individus capturés (i) et densité moyenne/ 101 (d) à $0,6 \mathrm{~m}$ et $1 \mathrm{~m}$ de profondeur pour les 19 espèces constantes.

Table 6. Hyporheic zone. Total number of animals collected (i) and average density/10 1 (d) at a depth of $0,6 \mathrm{~m}$ and $1 \mathrm{~m}$ for the 19 constant species.

\begin{tabular}{|c|c|c|c|c|c|c|}
\hline \multirow{2}{*}{\multicolumn{2}{|c|}{ Espèces }} & \multicolumn{2}{|c|}{$0,6 \mathrm{~m}$} & \multicolumn{2}{|c|}{$\mathrm{m}$} & \multirow{2}{*}{$\begin{array}{c}\text { U } \\
\text { Mann- } \\
\text { Whitney }\end{array}$} \\
\hline & & 1 & d & & & \\
\hline & Pf & 60 & $\overrightarrow{2,4}$ & 23 & 1,9 & n.s \\
\hline & Av & 722 & 15,0 & 334 & 7,6 & $\mathbf{s}$ \\
\hline & *As & 107 & 3,9 & 86 & 4,3 & n.s \\
\hline & D1 & 547 & 13,3 & 135 & 6,1 & n.s \\
\hline & ${ }^{*} \mathrm{Gr}$ & 1876 & 38,3 & 2033 & 42,3 & n.s \\
\hline & *Gsp & 7162 & 140,4 & 2575 & 52,6 & s \\
\hline \multirow{9}{*}{$\mathrm{H}$} & ${ }^{*} \overline{\mathrm{Ps}}$ & 275 & 7,8 & 202 & 6,5 & n.s \\
\hline & Ac & 71 & 3,7 & 23 & 1,4 & $\mathbf{s}$ \\
\hline & ${ }^{*} \mathrm{Cg}$ & 16 & 1,6 & 261 & 7,9 & $s$ \\
\hline & $\mathbf{B z}$ & 370 & 7,4 & 246 & 5,4 & n.s \\
\hline & $\mathrm{Be}$ & 748 & 14,9 & 456 & 9,7 & n.s \\
\hline & *Pd & 1090 & 24,2 & 135 & 5,0 & $\mathrm{~s}$ \\
\hline & *PV & 137 & 5,7 & 34 & 1,8 & s \\
\hline & *Pfm & 809 & 17,6 & 69 & 2,6 & $\mathbf{s}$ \\
\hline & ${ }^{*} \mathrm{Pn}$ & 121 & 3,9 & 95 & 3,0 & n.s \\
\hline$S$ & $* \mathrm{Vv}$ & 28 & 2,3 & 66 & 2,6 & n.s \\
\hline I & ${ }^{*} \mathrm{~Sv}$ & 53 & 1,9 & 83 & 2,6 & n.s \\
\hline \multirow[t]{2}{*}{$\mathrm{A}$} & ${ }^{*} \mathrm{Sg}$ & 147 & 11,3 & 163 & 6,0 & n.s \\
\hline & ${ }^{*}$ It & 34 & 2,8 & 8 & 1,1 & n.s \\
\hline
\end{tabular}

$P$. vandeli, $P$. f. meridionalis et épigées, $A$. venustus et $A$. crassa sont significativement plus abondantes à 0,6 qu'à $1 \mathrm{~m}$. Seule l'espèce stygobie, $C$. gallicus, est significativement plus abondante à $1 \mathrm{~m}$ qu'à $0,6 \mathrm{~m}$.

Au total, l'occupation du site par ces 19 espèces est caractérisée par une forte hétérogénéité. En effet, ces espèces peuvent se répartir en six catégories (Tableau 7), selon leur fréquence d'occurence et leur abondance à 0,6 et $1 \mathrm{~m}$ de profondeur.

\subsection{Le peuplement des Cyclopides}

\subsubsection{Caractéristiques générales}

Composé de 7 espèces, 3 stygobies et 4 épigées (tableau 1), ce peuplement est marqué par la dominance des formes hypogées $(88,2 \%$ du total des récoltes) dont Graeteriella sp. $(62,05 \%)$ et Graeteriella rouchi $(24,9 \%)$ sont les taxons les plus communs.

On a vu que l'abondance globale de chacune de ces espèces ne présente pas de différence significative lors des 13 séries de prélèvements. En
Tableau 7. Zone hyporhéique. Distribution des 19 espèces constantes selon leur occurence et leur abondance à $0,6 \mathrm{~m}$ et $1 \mathrm{~m}$ de profondeur.

Table 7. Hyporheic zone. Distribution of the 19 constant species according to their occurrence and abundance at a depth of $0,6 \mathrm{~m}$ and $1 \mathrm{~m}$.

\begin{tabular}{|c|c|c|c|}
\hline abondance & $0,6>1 \mathrm{~m}$ & $0,6=1 \mathrm{~m}$ & $1 \mathrm{~m}>0,6$ \\
\hline $0,6>1 \mathrm{~m}$ & $\begin{array}{l}\text { *P. dianae } \\
\text { *P. f. meri. }\end{array}$ & $\begin{array}{l}\text { *P. vandeli } \\
\text { A. crassa } \\
\text { *G. sp. } \\
\text { A. venustus }\end{array}$ & \\
\hline $0,6=1 \mathrm{~m}$ & $\begin{array}{l}\text { P. fimbriatus } \\
\text { D. languidoides }\end{array}$ & $\begin{array}{l}* \text { P. nertensis } \\
\text { *P. subterranea } \\
\text { B. echinatus } \\
\text { B. zschokkei } \\
\text { *G. rouchi } \\
\text { * A. sensitivus }^{*} \text {-S. virei } \\
\text { *I. thibaudi }\end{array}$ & $\begin{array}{l}\text { *V. vandeli } \\
{ }^{*} \text { S. gineti }\end{array}$ \\
\hline $1 \mathrm{~m}>0,6$ & & & ${ }^{*}$ C. gallicus \\
\hline
\end{tabular}

conséquence, il n'est pas étonnant que leur rang selon leur dominance au cours de ces 13 séries ne varie pas de façon significative (coefficient de concordance de rang de Kendall $W=0,909$ et $\chi^{2}$ $=70,9$ avec $\chi^{2} \cdot 0,001=22,46$ pour $6 \mathrm{dl}$ ).

De même, le rang de ces espèces selon leur fréquence d'occurence dans ces 13 séries de prélèvements ne montre pas de différence significative ( $W$ de Kendall $=0,881$ et $\chi^{2}=68,71$ avec $\chi^{2} 0,001$ $=22,46$ pour $6 \mathrm{dl}$ ).

En outre, fréquence d'occurence et dominance sont très significativement ou significativement correlées dans chaque série d'échantillons. Le coefficient de correlation de rang de Spearman rs, présente les valeurs suivantes : $0,934,0,991,0,963$, $0,981,0,927,0,763,0,926,0,963,0,807,0,972$, $0,954,0,991,0,991$ (rs $0,01=0,893$ et is 0,05 $=0,714$ pour $\mathrm{N}=7$ ).

\subsubsection{Structure du peuplement}

Une analyse factorielle des correspondances a été réalisée à partir des 100 échantillons contenant des Cyclopides. 6 des 7 espèces recensées ont été retenues; trois stygobies : Graeteriella sp, $G$. rouchi et $A$. sensitivus ; trois épigées : $A$. venustus, $P$. fimbriatus et $D$. languidoides. $E$. serrulatus, peu abondant et peu fréquent, a été éliminé.

Les trois premiers axes expriment $90,3 \%$ de la variance totale avec, pour valeurs respectives, $47,3 \%, 30,1 \%$ et $12,9 \%$. L'axe 1 est déterminé 
dans sa partie négative par $D$. languidoides et $A$. venustus, tous deux épigés, et dans sa partie positive, par G. rouchi (stygobie). L'axe 2 est défini par Graeteriella sp. (stygobie) dans sa partie négative et par $G$. rouchi dans sa partie positive. Cette structure est donc relativement simple (Fig. 7). L'affectation des 100 échantillons à ces trois groupements a été établie à l'aide d'une classification automatique (aggrégation autour de centres mobiles) réalisée à partir des coordonnées de ces échantillons dans l'AFC. Dans l'espace 1-2, le groupe G. rouchi occupe le quart supérieur droit; le groupe Graeteriella. sp., la zone centrale inférieure et le groupe
D. languidoides le quart supérieur gauche. Cette partition consiste à séparer les échantillons riches en formes hypogées (G.r. et $G$. sp.) de ceux où les espèces épigées (D.I. et $A . v$.), sans être dominantes, sont nettement plus abondantes (Tableau 8).

Reportés sur la carte du site, ces trois groupements présentent une configuration assez complexe (Fig. 8). Dans le secteur $A$ et les zones attenantes des secteurs $B$ et $C$, le peuplement est fortement dominé par Graeteriella sp. indépendamment de la profondeur. Vers l'aval du secteur $C$ où le drainage s'accentue, $G$. sp. est remplacée par $G$. rouchi qui

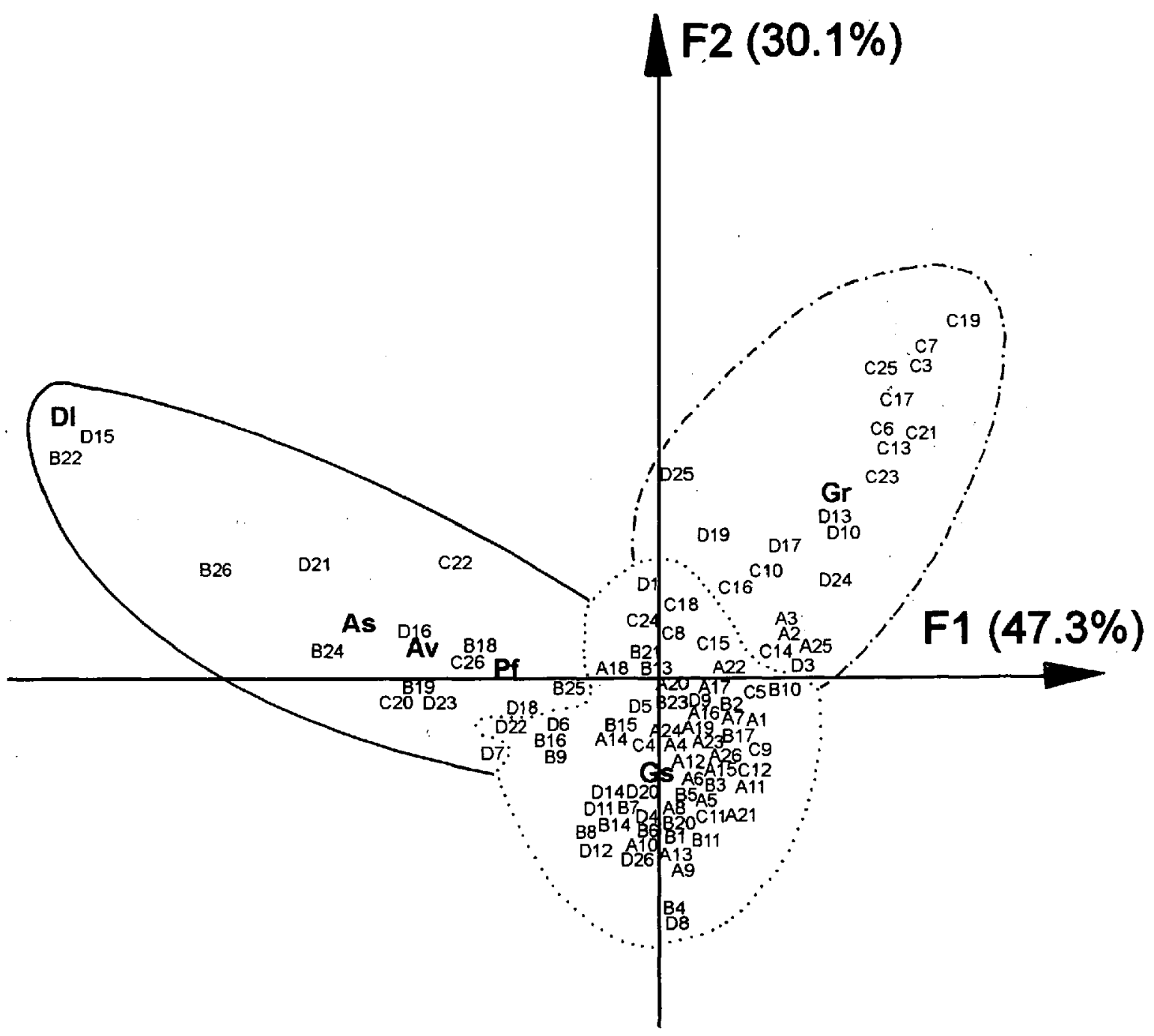

Fig. 7. Analyse factorielle des correspondances des échantillons de Cyclopides.

Fig. 7. Correspondence analysis of the cyclopoid samples. 


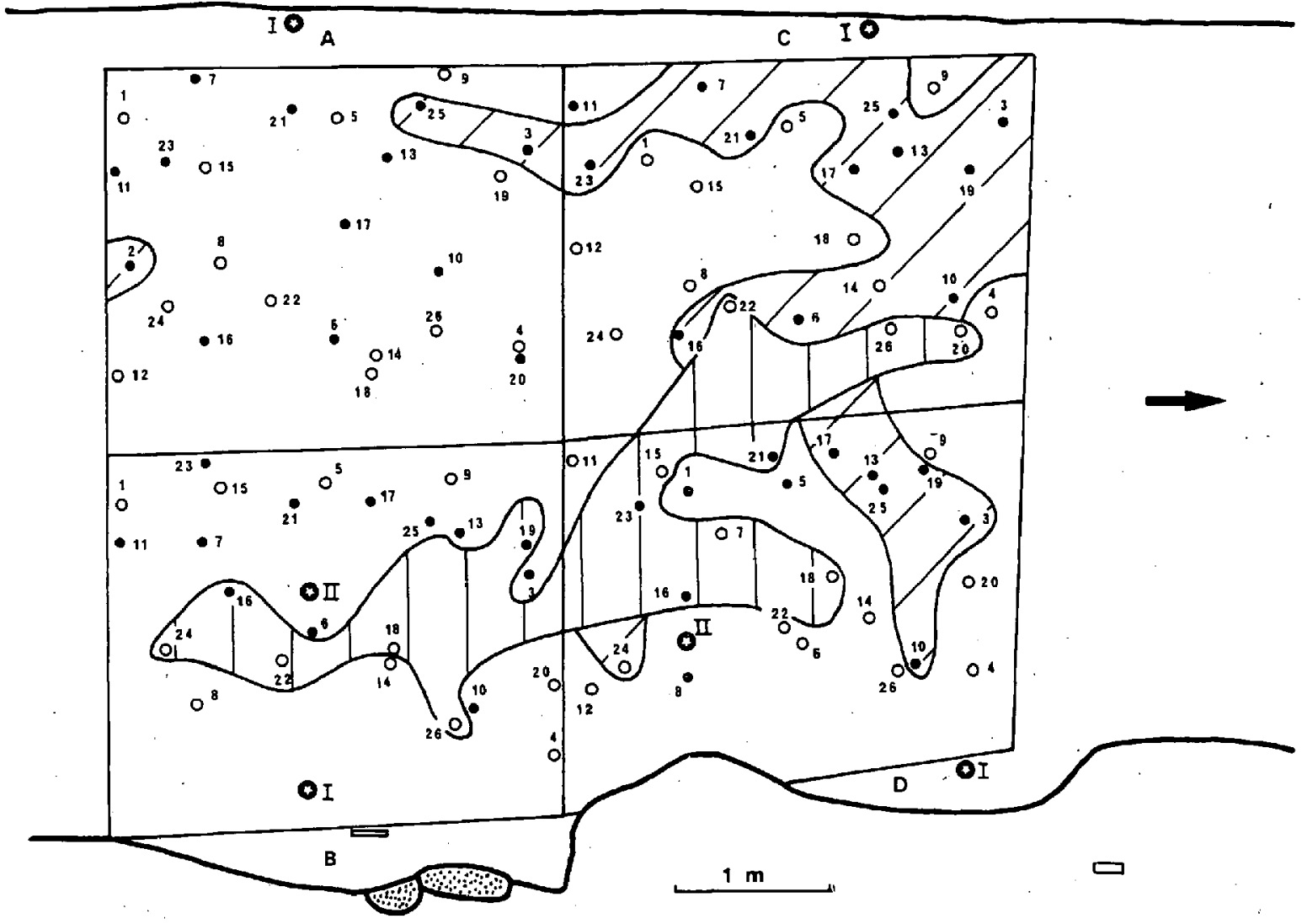

Fig. 8. Carte de distribution des trois groupements de Cyclopides mis en évidence par l'analyse factorielle des correspondances ; en blanc, groupe Graeteriella sp. ; lignes inclinées, groupe Graeteriella rouchi ; lignes verticales, groupe Diacyclops languidoides.

Fig. 8. Spatial distribution of the three cyclopoid groups as shown by correspondence analysis ; Graeteriella sp. group (white) ; Graeteriella rouchi group (oblique lines); Diacyclops languidoides group (vertical lines).

Tableau 8. Zone hyporhéique. Caractéristiques des trois groupements de Cyclopides. $n$ : nombre de stations ; i/10 1 : densité moyenne ; $\mathrm{H}^{\prime}$ : indice de diversité de Shannon-Weaver. Table 8. Hyporheic zone. Characteristics of the three cyclopoid groups. $\mathrm{n}$ : number of stations ; $\mathrm{i} / 10 \mathrm{l}$ : average density ; $\mathrm{H}^{\prime}$ : diversity index of Shannon-Weaver.

\begin{tabular}{|l|c|c|c|c|}
\hline Groupe & $\mathbf{n}$ & $\%$ hyp. & $\mathrm{i} / 10 \mathrm{I}$ & $\mathrm{H}^{\prime}$ \\
\hline G. species & 63 & 92,0 & 176 & 1,236 \\
\hline G. rouchi & 22 & 96,2 & 115 & 1,199 \\
\hline D. languidoides & 15 & 57,6 & 136 & 2,086 \\
\hline
\end{tabular}

devient dominante à $1 \mathrm{~m}$ de profondeur. Sous le chenal, en $B$, et à la périphérie du banc de graviers en $D, G$. sp. reste la forme dominante ; mais l'abondance relative des espèces épigées $D$. languidoides et $A$. venustus, jusqu'alors faible (moins de $6 \%$ ), augmente nettement $(21,7 \%$ et $18,9 \%)$. Sous le chenal et ài l'aval de l'axe de drainage du secteur $D$, $G$. sp. et $G$. rouchi sont, à nouveau, les formes dominantes avec la même discrimination selon la profondeur et la position par rapport au drainage que dans les secteurs $A$ et $C$. Dans cette bande étroite, proche de la rive droite, les densités s'effondrent puisque toutes espèces confondues elles sont de l'ordre de $70 \mathrm{i} / 101$ en moyenne.

Le groupe $D$. languidoides est celui qui possède l'indice de diversité de Shannon-Weaver lè plus élevé $\left(\mathrm{H}^{\prime}=2,086\right)$. Les groupes Graeteriella sp. $\left(\mathrm{H}^{\prime}=\right.$ $1,236)$ et $G$. rouchi $\left(H^{\prime}=1,199\right)$, marqués par la forte dominance de ces espèces, ont un indice nettement plus faible. 


\subsection{Le peuplement des Harpacticides}

\subsubsection{Caractéristiques générales}

Composé de 16 espèces, 8 stygobies et 8 épigées (Tableau 1), ce peuplement est dominé par les formes hypogées $(63,2 \%$ du total des récoltes).

On a vu que les densités globales des 9 espèces les plus abondantes, à l'exception de $B$. zschokkei, ne montrent pas de différence significative lors des 13 séries de prélèvements. En conséquence, le rang de ces espèces selon leur abondance dans les 13 séries de prélèvements ne varie pas de façon significative (coefficient de concordance de rang de Kendall W $=0,787$ et $\chi^{2}=81,84$ avec $\chi^{2} 0,001=26,12$ pour $8 \mathrm{dl}$ ). De même, le rang de ces espèces selon leur fréquence d'occurrence dans les 13 séries de prélèvement ne montre pas de différence significative (W de Kendall $=0,703$ et $\chi^{2}=73,11$ avec $\chi^{2} 0,001=$ 26,12 pour $8 \mathrm{dl}$ ).

Enfin, pour ces 9 espèces, fréquence d'occurrence et dominance sont très significativement ou significativement corrélées dans 11 séries d'échantillons sur 13 ; le coefficient de corrélation de rang de Spearman rs, présente les valeurs suivantes : $0,903,0,856$, $0,655,0,895,0,525,0,872,0,690,0,809,0,883$, $0,720,0,510,0,749,0,629$ (rs $0,01=0,783$ et rs 0,05 $=0,600$ pour $\mathrm{N}=9$ ).

\subsubsection{Structure du peuplement}

Une AFC a été réalisée à partir des 103 échantillons contenant des Harpacticides, 10 espèces ont été retenues : 7 stygobies, $P$. dianae, $P$. $f$. meridionalis, $P$. nertensis, $P$. vandeli, $P$. subterranea, $N$. gracilis, $C$. gallicus, 3 épigées, $B$ (L.) echinatus, B. zschokkei, A. crassa (Fig. 9).

Les trois premiers axes expriment $60,5 \%$ de la variance totale avec pour valeurs respectives $(25,1 \%, 19,1 \%, 16,3 \%)$. L'axe 1 est déterminé dans sa partie négative par $P$. dianae et $P$. fontinalis meridionalis, toutes deux stygobies, et par $B(L$. echinatus (épigée) dans sa partie positive. L'axe 2 est caractérisé par $C$. gallicus et $P$. subterranea, toutes deux stygobies, dans sa partie positive et par $B$ (L.) echinatus dans sa partie négative. L'axe 3 est défini par deux espèces stygobies, $P$. vandeli dans sa partie positive, $P$. subterranea dans sa partie négative.

L'axe 1 oppose donc des prélèvements riches en Parastenocaris $(P$. dianae et $P$. fontinalis meridionalis) à une double série d'échantillons discriminés par l'axe 2 : ceux riches en formes épigées $(B(L$.)e, $B z, A c$ ) et ceux riches en $C$. gallicus et $P$. subterranea, espèces stygobies. L'axe 3 permet d'individualiser une dernière catégorie d'échantillons, caractérisés par l'espèce hypogée $P$. vandeli. L'affectation des 103 échantillons à ces quatre groupements a été établie à l'aide d'une classification automatique (aggrégation autour de centres mobiles) réalisée à partir des coordonnées des échantillons dans l'AFC.

Dans l'espace 1-2, ces groupements occupent schématiquement les positions suivantes : groupe $P$. dianae, quart inférieur gauche ; groupe $B(L$.$) echina-$ tus, quart inférieur droit ; groupe $C$. gallicus, quart supérieur droit ; groupe $P$. vandeli, quart supérieur gauche.

Reportés sur la carte du site, ces quatre groupements forment une série de bandes longitudinales (Fig. 10).

Le groupe $B$ (L.) echinatus s'étend le long de la rive droite et rassemble des échantillons issus des horizons $0,6(12)$ et $1 \mathrm{~m} \mathrm{(7)}$.

Le groupe $C$. gallicus longe le précédent. Les échantillons prélevés à $1 \mathrm{~m}$ y sont majoritaires (16 pour 3 seulement à $0,6 \mathrm{~m}$ ).

Le groupe $P$. dianae occupe la zone médiane ainsi qu'une bande étroite dans le secteur $D$. Il rassemble des échantillons essentiellement prélevés à $0,6 \mathrm{~m}$ (27 pour 3 seulement à $1 \mathrm{~m}$ ).

Le groupe $P$. vandeli s'étend le long de la rive gauche. Il est indistinctement composé d'échantillons provenant de $0,6 \mathrm{~m}$ (9) et de $1 \mathrm{~m}$ (12).

L'homogénéité de l'aire de ce dernier groupe est rompue par la présence de 14 échantillons appartenant aux trois autres groupements. Sauf $\mathrm{Cl}$, tous ces prélèvements proviennent de $1 \mathrm{~m}$ de profondeur ; 9 d'entre eux appartiennent au groupe $C$. gallicus en raison de l'importance de cette espèce à cette profondeur.

Ainsi, le peuplement des Harpacticides de ce site suit une double zonation, horizontale et verticale. Dans le banc de gravier, le peuplement est dominé par les formes hypogées ; les valeurs de l'indice de diversité H' y sont relativement élevées (Tableau 9). Sous le chenal les espèces épigées l'emportent ; $\mathrm{H}^{\text {' }}$ est nettement plus faible. La densité moyenne des individus de ces quatre groupements est également variable puisque de la rive gauche à la rive droite, 
F2 (19.1\%)

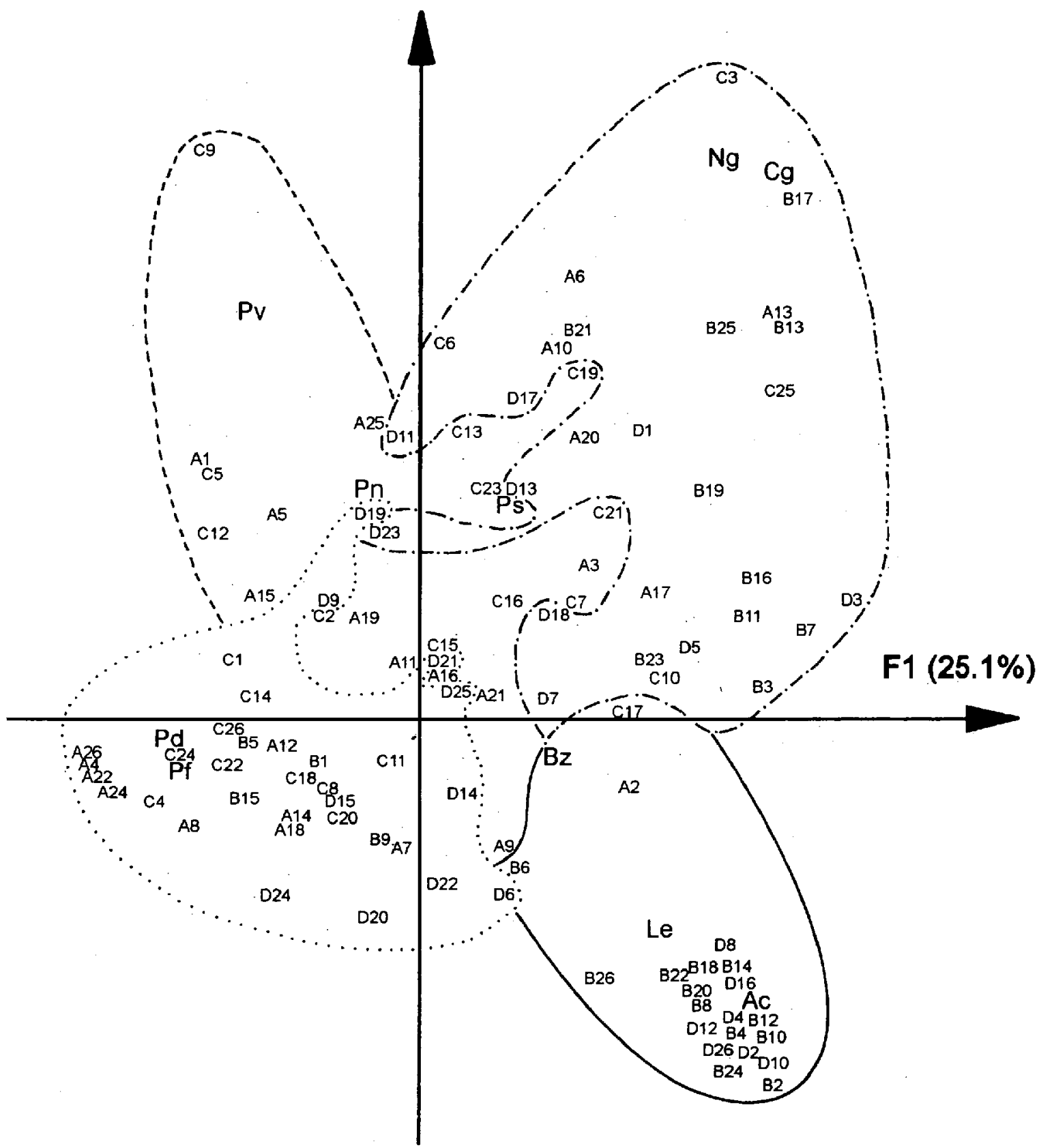

Fig. 9. Analyse factorielle des correspondances des échantillons d'Harpacticides.

Fig. 9. Correspondence analysis of the harpacticoid samples. 


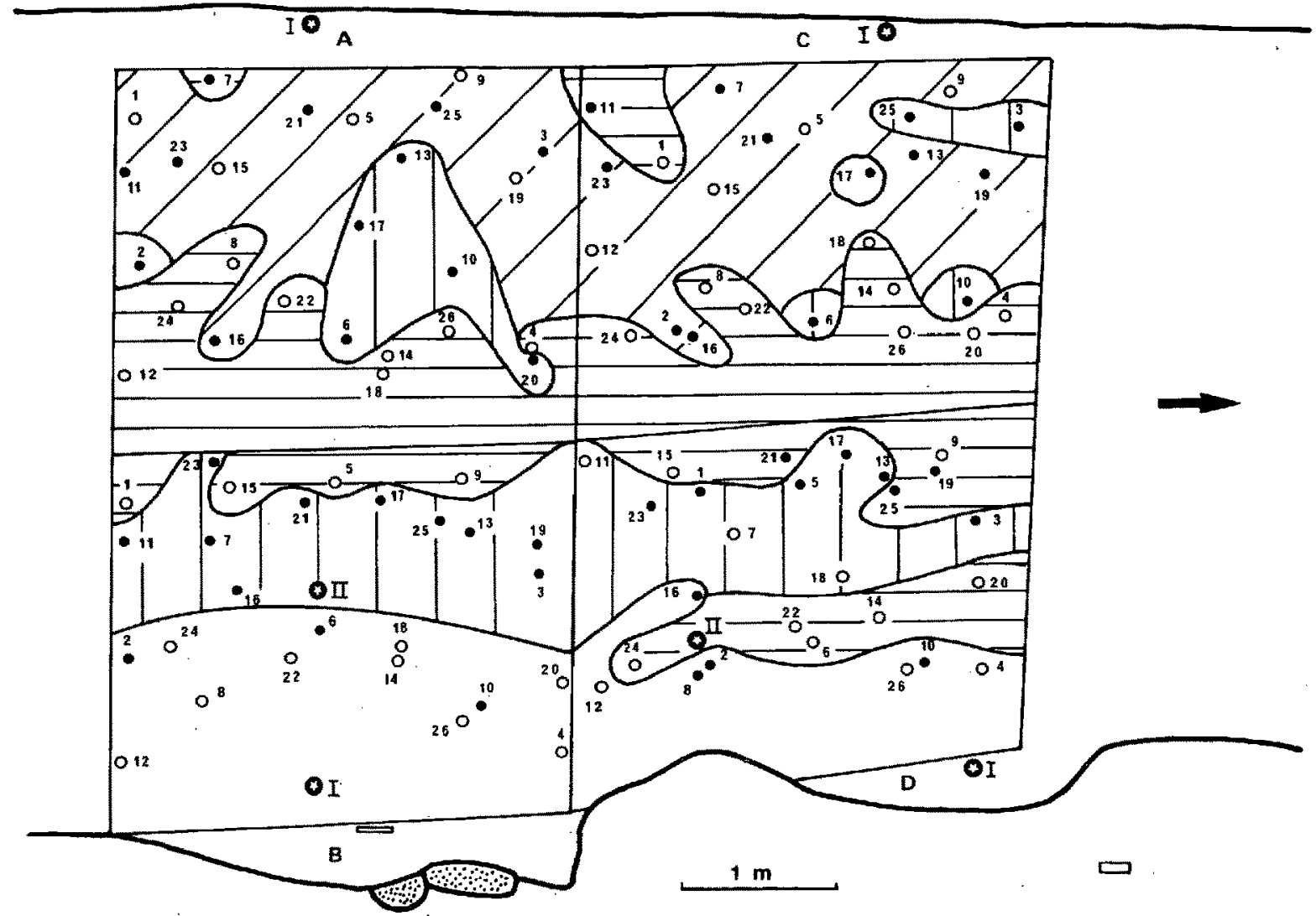

Fig. 10. Carte de la distribution des quatre groupements d'Harpacticides mis en évidence par l'analyse factorielle des correspondances ; en blanc, groupe Bryocamptus ( L.) echinatus; lignes verticales, groupe Ceuthonectes gallicus; lignes horizontales, groupe Parastenocaris dianae ; lignes inclinées, groupe Parastenocaris vandeli.

Fig. 10. Spatial distribution of the four harpacticoid groups as shown by correspondence analysis ; Bryocamptus (L.) echinatus group (white); Ceuthonectes gallicus group (vertical lines) ; Parastenocaris dianae group (horizontal lines) ; Parastenocaris vandeli group (oblique lines).

Tableau 9. Zone hyporhéique. Caractéristiques des quatre groupements d'Harpacticides. $n$ : nombre de stations ; $i / 101$ : densité moyenne ; $H^{\prime}$ : indice de diversité de ShannonWeaver.

Table 9. Hyporheic zone. Characteristics of the four harpacticoid groups. $n$ : number of stations; $i / 101$ : average density ; $H^{\prime}$ : diversity index of Shannon-Weaver.

\begin{tabular}{|l|c|c|c|c|}
\hline Groupe & n & \% hypo. & i/10 l & H $^{\prime}$ \\
\hline P. vandeli & 21 & 71,7 & 28 & 2,915 \\
\hline P. dianae & 33 & 77,3 & 80 & 2,305 \\
\hline C. gallicus & 28 & 66,4 & 40 & 2,725 \\
\hline B. echinatus & 21 & 12,6 & 43 & 1,915 \\
\hline
\end{tabular}

elle présente les valeurs suivantes $=28-80-40-$ $43 \mathrm{i} / 10 \mathrm{l}$.

Cette zonation longitudinale du peuplement des Harpacticides se superpose globalement à celle de la perméabilité et de la piézométrie.

\section{Discussion et conclusions}

L'étude du peuplement des Crustacés de la zone hyporhéique d'un ruisseau des Pyrénées, le Nert, a été réalisée sur une zone de $30 \mathrm{~m}^{2}$, soit à un niveau d'échelle métrique à décimétrique.

\subsection{Richesse spécifique}

Ce peuplement, composé d'Ostracodes, Copépodes, Syncarides, Isopodes et Amphipodes (les 
Cladocères sont absents), a une richesse spécifique totale de 35 espèces (20 stygobies). Bien qu'inférieure à celle observée (Rouch 1988) dans un ruisseau voisin, le Lachein (50 espèces dont 22 stygobies), cette richesse spécifique n'en demeure pas moins élevée, notamment celle des Copépodes (23 espèces), si on la compare à celle mise en évidence pour d'autres cours d'eau lors de travaux menés à la même échelle (Bou 1979, Dole 1983, Whitman \& Clark 1984, Pennak \& Ward 1986, Bretschko \& Liechtfried 1988, Strayer 1988, Strommer \& Smock 1989, Williams 1989, Boulton et al. 1992, Cooling \& Boulton 1993). Le fort pourcentage des espèces stygobies $(57 \%$ des taxọs recensés) différencie également le milieu hyporhéique du Nert des autres sites étudiés.

Si la richesse globale des Crustacés du milieu hyporhéique du Nert s'élève à 35 espèces, elle n'atteint que 20 à 29 espèces lors de chaque série mensuelle de 8 prélèvements et n'est plus que de 12 espèces en moyenne par prélèvement. Ce résultat implique que les recherches faunistiques de type extensif sous-estiment vraisemblablement le nombre réel de formes stygobies vivant dans la zone hyporhéique.

\subsection{Abondance}

Dans le milieu hyporhéique du Nert, les Copépodes constituent le groupe le plus abondant $(96,8 \%$ des récoltes) et, notamment, les Cyclopides $(72,7 \%)$. La densité moyenne des Crustacés y est de $279 \mathrm{i} / 10 \mathrm{l}$ à $-0,6 \mathrm{~m}$ et de $139 \mathrm{i} / 10 \mathrm{l}$ à $-1 \mathrm{~m}$. Ces valeurs sont nettement inférieures à celles observées par Pennak \& Ward (1986). Elles sont comparables à celles du ruisseau de Lachein (Rouch 1988) et supérieures à celles indiquées par Gibert et al. (1977) et Dole (1983) pour la station 9 du canal de Miribel ou par Danielopol (1976) pour la station March du Danube.

Les espèces stygobies représentent $82,2 \%$ des individus récoltés ; leur abondance relative globale ne varie pas aux deux profondeurs étudiées. Ce résultat confirme le propos de Danielopol (1991) selon lequel les espèces hypogées peuvent surpasser en nombre les formes épigées dans ce type de milieu superficiel.

Ce fort pourcentage d'hypogées pourrait parâtre d'autant plus paradoxal que la zone étudiée a toujours été caractérisée par un gradient hydraulique négatif (zone de recharge de la nappe). Or, lorsque les échanges ont lieu depuis la surface vers la zone hyporhéique (downwelling), ils entraînent en profondeur la faune épigée (Marmonier \& Dole 1986, Marmonier 1988, Marmonier \& Creuzé des Châtelliers 1991, Dole-Olivier \& Marmonier 1992). En fait, l'étude hydrologique de ce site (Rouch et al., sous presse) a montré que la dynamique des échanges n'était pas de type vertical direct mais relevait davantage du type « substream» (Harvey \& Bencala 1993) selon un caractère longitudinal très marqué. En conséquence, l'entraînement vers la profondeur des espèces épigées ne semble pas un phénomène important sur le Nert.

\subsection{Persistance}

Sur le site étudié, ni l'abondance de l'ensemble des Crustacés, ni celle des espèces constantes (excepté $B$. zschokkei) ne présentent des différences significatives lors des 13 séries de prélèvements. Le rang des espèces selon leur abondance ne montre pas, non plus, de différence significative. En outre, la composition faunistique reste très stable dans le temps. Rahel (1990) utilise le terme de persistance pour qualifier la constance de telles communautés vérifiée au niveau de l'abondance absolue, des rangs d'abondance et de la présence-absence des espèces. Cet auteur ajoute que la persistance indique une organisation des communautés plutôt déterministe que stochastique. Cette stabilité du peuplement, si elle est en accord avec les données établies pour le ruisseau de Lachein (Rouch 1988, 1991) va à l'encontre de la plupart des résultats concernant l'hyporheos permanent : Copépodes (Williams \& Hynes 1974, Whitman \& Clark 1984, Pennak \& Ward 1986, Strayer 1988, Palmer 1990), Ostracodes (Marmonier 1984, 1988, Danielopol 1984, 1989). Toutefois, Giberson \& Hall (1988) n'observent pas de variations saisonnières des invertébrés autres que les insectes dans un cours d'eau canadien ; Kowarc (1991) pour les Harpacticides du Seebach et Creuzé des Châteliers \& Marmonier (1993) pour les Ostracodes de Miribel et Bregnier sur le Rhône font le même type d'observation.

La persistance observée sur le Nert implique donc que la communauté des Crustacés de ce site n'est pas influencée par les perturbations de l'environnement. Parmi ces dernières, les crues sont considérées comme un des facteurs essentiels de déstabilisation du milieu hyporhéique (Williams \& Hynes 1974, Danielopol 1976, Marmonier \& Dolé 1986, Marmonier 1988, 1991, Strommer \& Smock 1989, 
Marmonier \& Creuzé des Châtelliers 1991, Dole et Marmonier 1992, Palmer 1990, Palmer et al. 1992). Sur le Nert, lors de l'année étudiée, comme sur le Lachein (Rouch 1988), les crues n'ont modifié ni la morphologie du lit, ni la structure globale des écoulements interstitiels (Rouch 1992, Rouch et al. sous presse) ni la structure des peuplements. Ce résultat incite à penser que la zone hyporhéique pourrait, dans certains cours d'eau, être considérée comme stable à une échelle vraisemblablement pluriannuelle.

\subsection{Hétérogénéité spatiale}

L'hétérogénéité spatiale du peuplement des Crustacés de la zone hyporhéique du Nert se vérifie dans la répartition et l'abondance des espèces selon les plans horizontal et vertical. La distribution des groupements de Copépodes est très caractéristique à cet égard. Elle est liée aux caractères morphologiques (opposition banc - chenal) et hydrogéologiques (opposition des zones de drainage avec une bonne perméabilité à celles moins bien drainées avec une perméabilité plus faible).

On a vu que, sur le Nert, l'hydrologie de surface n'intervenait pas sur la structure temporelle du peuplement interstitiel. Elle n'en joue pas moins un rôle important en déterminant les caractéristiques morphologiques du site étudié, banc et chenal, dont les peuplements sont globalement différents. Dans ce cadre morphologique, l'organisation des écoulements souterrains provoque une différentiation supplémentaire. L'influence de la dynamique des écoulements sur le peuplement hyporhéique, pressentie par Angelier (1953, 1962) est maintenant bien établie (Godbout \& Hynes 1982, Marmonier \& Dole 1986, Marmonier 1985, 1988, Rouch 1988, 1992, Bretschko \& Leichtfried 1988, Creuzé des Châtelliers \& Reygrobellet 1990, Creuzé des Châtelliers 1991, Danielopol 1991, Boulton et al. 1992, Stanley \& Boulton 1993).

Dole-Olivier \& Marmonier (1992) ont montré que lorsque les caractéristiques hydrogéologiques et géomorphologiques sont superposées, elles déterminent une structure précise du peuplement. Les résultats du Nert confirment le bien-fondé de cette observation. Ils montrent, en outre, que de telles structures peuvent se perpétuer dans le temps en liaison avec la stabilité de l'habitat.
L'hétérogénéité de la distribution des formes stygobies dans le milieu hyporhéique du Nert conduit à nouveau (Rouch 1986, Dole \& Marmonier 1992, Dole-Olivier et al. 1993, Danielopol 1991, Danielopol \& Rouch 1991) à souligner la diversité des potentialités écologiques des espèces stygobies et, de ce fait, la diversité de leurs adaptations.

\section{Remerciements}

Je remercie A. Pitzalis, A. Mangin, M. Bakalowicz et C. Andrieux pour l'aide qu'ils m'ont apportée sur le terrain, A. Descouens qui a effectué la totalité du tri des récoltes ainsi que D. D'Hulst pour la partie informatique. F. Boineau a saisi le manuscrit.

\section{Travaux cités}

Angelier E. 1953. - Recherches écologiques et biogéographiques sur la faune des sables submergés. Arch. Zool. exp. gén., 90 : 37-161.

Angelier E. 1962. - Remarques sur la répartition de la faune dans le milieu interstitiel hyporhéique. Zool. Anz., 168 : 351-356.

Barrouquère G.. 1968. - Structure des formations paléozoïques du massif de l'Arize (Pyrénées ariégeoises). Bull. B.R.G.M., $2: 1-10$.

Bou C. 1979. - Etude de la faune interstitielle des alluvions du Tarn. Mise en place d'une station d'étude et résultats préliminaires. Bull. Féd. Tarn spéléo. Archéol., 16 : 117-129.

Bou C. \& Rouch R. 1967. - Un nouveau champ de recherches sur la faune aquatique souterraine. C.R. Açad. Sci. Paris, $265: 369-370$.

Boulton A.J., Valett H.M. \& Fisher S.G. 1992. - Spatial distribution and taxonomic composition of the hyporheos of several Sonoran Desert streams. Arch. Hydrobiol., 125: $37-61$.

Bretschko G. 1991. - Bed sediments, groundwater and stream limnology. Verh. Internat. Verein. Limnol., 24 : 1957-1960.

Bretschko G. \& Leichtfried M. 1988. - Distribution of organic matter and fauna in a second order, alpine gravel stream (Ritrodat-Lunz study area, Austria). Verh. Internat. Verein. Limnol., 23 : 1333-1339.

Cooling M.P. \& Boulton A.J. 1993. - Aspects of the hyporheic zone below the terminus of a south australian arid-zone stream. Aust. J. Mar. Freshwater Res., 44 : 411-426.

Creuzé des Châtelliers M. 1991. - Dynamique de répartition des biocénoses interstitielles du Rhône en relation avec des caractéristiques géomorphologiques. Thèse doc. Univ. Lyon, 1, $161 \mathrm{p}$.

Creuzé des Châtelliers M. \& Reygrobellet J.L. 1990. - Interactions between geomorphological processes, benthic and hyporheic communities : first results on a by-passed canal of the french upper Rhône river. Regulated rivers : Research \& Management, 5 : 139-158.

Creuzé des Châtelliers M. \& Marmonier P. 1993. - Ecology of benthic and interstitial ostracods of the Rhône-river, France. Journal of Crustacean Biology, 13 : 268-279.

Danielopol D. 1976. - The distribution of the fauna in the interstitial habitat of riverine sediments of the Danube and the Piesting (Austria). Int. J. Speleol;; 8 : 23-51. 
Danielopol D. 1983. - Der Einfluss organischer Verschmutzung auf das Grundwasser-Ökosystem der Donau im Raum Wien und Niederösterreich. Bundesministerium f. Gesundheit $u$. Umweltschutz, Wien, Forschungsberichte, 5: 5-160.

Danielopol D. 1989. - Groundwater fauna associated with riverine aquifers. J.N. Am. benth. Soc., $8:$ 18-35.

Danielopol D. 1991. - Spatial distribution and dispersal of interstitial Crustacea in alluvial sediments of a backwater of the Danube at Vienna. Stygologia, $6: 97-110$.

Danielopol D. \& Rouch R. 1991. - L'adaptation des organismes au milieu aquatique souterrain. Réflexions sur l'apport des recherches écologiques récentes. Stygologia, 6 : 129-142.

Danielopol D. \& Marmonier P. 1992. - Aspects of research on groundwater along the Rhône, Rhine and Danube. Regulated rivers : Research \& Management, $7: 5-16$.

Dole M.J. 1983. - Le domaine aquatique souterrain de la plaine alluviale du Rhône à l'est de Lyon ; écologie des niveaux supérieurs de la nappe. Thèse de $3^{\mathrm{e}}$ cycle, Univ. Claude Bernard, Lyon I : $168 \mathrm{p}$.

Dole M.J. \& Chessel D. 1986. - Stabilité physique et biologique des milieux interstitiels. Cas de deux stations du HautRhône. Annls Limnol., 22 : 68-81.

Dole-Olivier M.J. \& Marmonier P. 1992. - Patch distribution of interstitial communities : prevailing factors. Freshwater Biology, 27 : 177-191.

Dole-Olivier M.J., Creuzé des Châtelliers M. \& Marmonier P. 1993. - Repeated gradients in subterranean landscapes. Example of the stygofauna in the alluvial floodplain of the Rhône River (France). Arch. Hydrobiol., 127 : 451-471.

Gibert J., Ginet R., Mathieu J., Reygrobellet J.L. \& SeyedReihani A. 1977. - Structure et fonctionnement des écosystèmes du Haut-Rhône français. IV. Le peuplement des eaux phréatiques ; premiers résultats. Annls Limnol., 13 : 83-97.

Gibert J., Ginet R., Mathieu J, \& Reygrobellet J.L. 1981. Structure et fonctionnement des ecosystèmes du Haut-Rhône français. IX. Analyse du peuplement de deux stations phréatiques alimentant des bras morts. Int. J. Speleol., 11 : 141-158.

Gibert J., Dole-Olivier M.J., Marmonier P. \& Vervier P. 1990. - Surface water - groundwater ecotones. The ecology and management of aquatic-terrestrial ecotones (R.J. Naiman \& H. Décamps eds), pp. 199-225. Man and the Biosphere series, UNESCO, Paris \& Parthenon Publishing group, Carnforth.

Giberson D.J. \& Hall R.J. 1988. - Seasonal variation in faunal distribution within the sediments of a canadian shield stream, with emphasis on responses to spring floods. Can J. Fish. Aquat. Sci., 45 : 1994-2002.

Godbout L. \& Hynes H.B.N. 1982. - The three dimensional distribution of the fauna in a single riffle in a stream in Ontario. Hydrobiologia, $97: 87-96$.

Gourbault N. \& Lescher-Moutoué F. 1968. - Recherches sur les eaux souterraines. 4. Etude de la faune hypogée peuplant le sous-écoulement du Nert. Ann. Spéléol., 23 : 735-742.

Harvey J.W. \& Bencala K.E. 1993. - The effect of streambed topography on surface - subsurface water exchanges in mountain catchments. Water Resour. Res., 29 : 89-98.

Kowarc V. 1991. - Distribution of harpacticoids in a second order mountain stream (Ritrodat-Lunz study area, Austria). Verh. Internat. Verein Limnol., 24 : 1930-1933.

Marmonier P. 1984. - Vertical distribution and temporal evolution of the ostracod assemblage of the Seebach sediments (Lunz, Austria). Jber. Biol. stn. Lunz., 7 : 49-82.
Marmonier P. 1985. - Répartition spatiale des Ostracodes dans les sédiments d'un ruisseau alpin (le Seebach, à Lunz, Autriche). Verh. Internat. Verein. Limnol,, 22 : 2053-2057.

Marmonnier P. 1988. - Biocénoses interstitielles et circulation des eaux dans le sous-écoulement d'un chenal aménagé du Haut-Rhône français. Thèse doc. Univ. Lyon I, 2 tomes, $161 \mathrm{p}$ et $108 \mathrm{p}$.

Marmonnier P. 1991. - Effect of alluvial shift on the spatial distribution of interstitial fauna. Verh. Internat. Verein. Limnol., 24 : 1613-1616.

Marmonnier P. \& Dole M.J. 1986. - Les Amphipodes des sédiments d'un bras court-circuité du Rhône. Sciences de l'eau, $5: 461-486$.

Marmonnier P. \& Creuzé des Châtelliers M. 1991. - Effects of spates on interstitial assemblages of the Rhône River. Importance of spatial heterogeneity. Hydrobiologia, $210: 243-251$.

Marmonier P., Dole-Olivier M.J. \& Creuzé des Châtelliers M. 1992. - Spatial distribution of interstitial assemblages in the floodplain of the Rhône River. Regulated rivers : Research \& Management, $7: 75-82$.

Palmer M.A. 1990. - Temporal and spatial dynamics of meiofauna within the hyporheic zone of Goose Creek, Virginia. J.N. Am. Benthol. Soc., 9 : 17-25.

Palmer M.A., Bely A.E. \& Berg K.E. 1992. - Response of invertebrates to lotic disturbance : a test of the hyporheic refuge hypothesis. Oecologia, $89: 182-194$.

Pennak R.W.\& Ward J.V. 1986. - Interstitial faunal communities of the hyporheic and adjacent groundwater biotopes of a Colorado mountain stream. Arch. Hydrobiol. Suppl. 74, 3 : 356-396.

Pospisil P. 1992. - Sampling methods for groundwater animals of unconsolidated sediments. The natural history of Biospeleology (A.I. Camacho ed.), pp. 107-134. Monografias del Museo Nacional de Ciencias Naturales, C.S.I.C., Madrid:

Rahel F.J. 1990. - The hierarchical nature of community persistence : a problem of scale. The American Naturalist, 136 : 328-344.

Rouch R. 1988. - Sur la répartition spatiale des Crustacés dans le sous-écoulement d'un ruisseau des Pyrénées. Annls Limnol., 24 : 213-234,

Rouch R. 1991 . - Structure du peuplement des Harpacticides dans le milieu hyporhéique d'un ruisseau des Pyrénées. Annls Limnol., 27 : 227-241.

Rouch R. 1992. - Caractéristiques et conditions hydrodynamiques des écoulements dans les sédiments d'un ruisseau des Pyrénées. Stygologia, 7 : 13-25.

Rouch R., Mangin A., Bakalowicz M. \& D'Hulst D. - The hyporheic zone : hydrogeological and geochimical study of a stream in the Pyrenees Mountains. Sous presse.

Stanford J.A. \& Gaufin A.R. 1974. - Hyporheic communities of two Montana rivers. Science, $185: 700-702$.

Stanford J.A. \& Ward J.V. 1988. - The hyporheic habitat of river ecosystems. Nature, 335 : 64-66.

Stanley E.H. \& Boulton A.J. 1993. - Hydrology and the distribution of hyporheos : perspectives from a mesic river and a desert stream. J.N. Am. Benthol. Soc., $12: 79-83$.

Strayer D. 1988. - Crustaceans and mites (Acari) from hyporheic and other underground waters in southeastern NewYork. Stygologia, $4:$ 192-207. 
Strommer J.L. \& Smock L.A. 1989. - Vertical distribution of invertebrates within the sandy substrate of a low-gradient headwater stream. Freshwater Biology, 22 : 263-274.

Whitman R.L. \& Clark W.J. 1984. - Ecological studies of the sand-dwelling community of an East-Texas stream. Freshwat. Invertebr. Biol., 5 : 59-79.

Williams D.D. 1984. - The hyporheic zone as a habitat for aquatic insects and associated arthropods. The ecology of aquatic insects (V. H. Resh and D.M. Rosenberg, Eds), pp. 430-455. Praeger Scientific, New York.
Williams D.D. 1989. - Towards a biological and chemical definition of the hyporheic zone in two canadian rivers. Freshwater Biology, 22 : 189-208.

Williams D.D. \& Hynes H.B.N. 1974. - The occurence of benthos deep in the substratum of a stream. Freshwater Biology $4: 233-256$. 